LA-UR-98 $\quad 2769$

\begin{tabular}{l|l} 
Title: & $\begin{array}{l}\text { SIMPLE RELATIONSHIPS FOR ESTIMATING } \\
\text { INTRAPARTICLE TRANSPORT EFFECTS FOR } \\
\text { CATALYTICALLY PROMOTED ENDOTHERMIC } \\
\text { REACTIONS }\end{array}$
\end{tabular}

Author(s):

Lee F. Brown

Engineering Science and Applications Division

Submitted to:

Informal Distribution for Review

MASTER

DISTRIEUTION OF THS DOCUMENT IS LNLIMTED

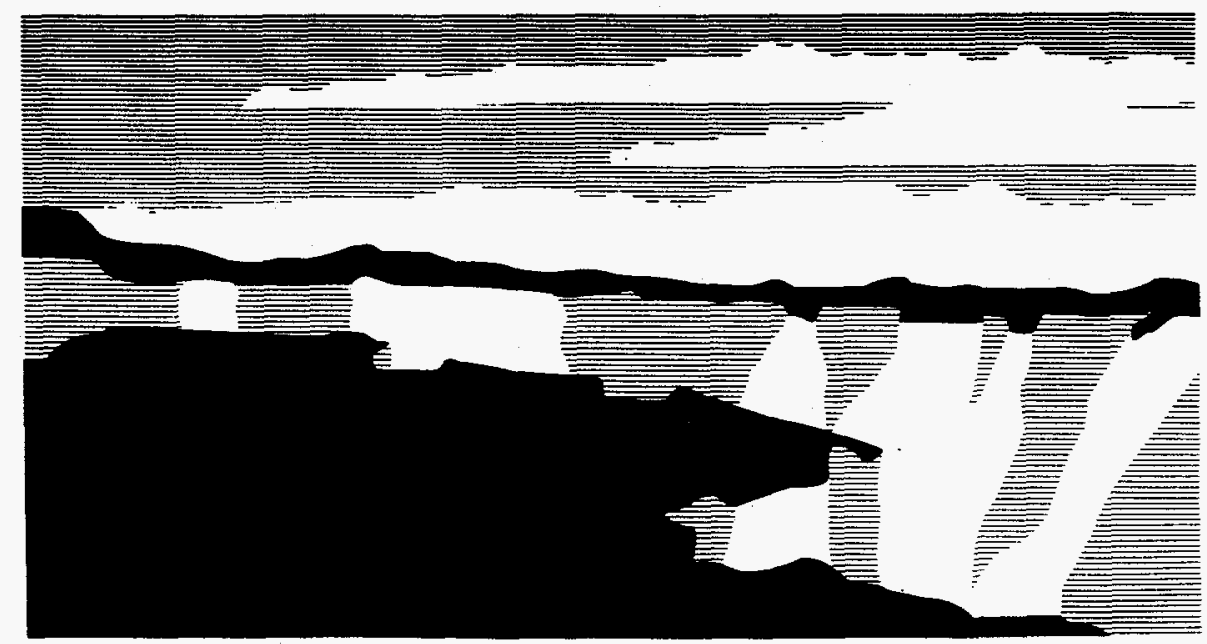

Los Alamos National Laboratory, an affirmative action/equal opportunity employer, is operated by the University of California for the U.S. Department of Energy under contract W-7405-ENG-36. By acceptance of this article, the publisher recognizes that the U.S. Government retains a nonexclusive, royalty-free license to publish or reproduce the published lorm of this contribution, or to allow others to do so, for U.S. Government purposes. The Los Alamos National Laboratory requests that the publisher identity this article as work performed under the auspices of the U.S. Department of Energy. 


\section{DISCLAIMER}

This report was prepared as an account of work sponsored by an agency of the United States Government. Neither the United States Government nor any agency thereof, nor any of their employees, makes any warranty, express or implied, or assumes any legal liability or responsibility for the accuracy, completeness, or usefulness of any information, apparatus, product, or process disclosed, or represents that its use would not infringe privately owned rights. Reference herein to any specific commercial product, process, or service by trade name, trademark, manufacturer, or otherwise does not necessarily constitute or imply its endorsement, recommendation, or favoring by the United States Government or any agency thereof. The views and opinions of authors expressed herein do not necessarily state or reflect those of the United States Government or any agency thereof. 


\section{DISCLAIMER}

Portions of this document may be illegible in electronic image products. Images are produced from the best available original document. 


\section{CONTENTS}

LIST OF TABLES $\quad$ ii

NOMENCLATURE $\quad$ iii

ACRONYMS iv

GLOSSARY $\quad$ iv

ABSTRACT 1

I. EXECUTIVE SUMMARY 1

II. EFFECTIVENESS FACTORS 3

A. Calculation and Estimation of Effectiveness Factors 3

B. Diagnostic Criteria for Mass and Heat Transfer Limitations 4

C. Scope of this Work $\quad 5$

III. THEORETICAL DEVELOPMENT

A. Effectiveness Factor of a Spherical Catalyst Particle

B. An Approximation to the Temperature Profile 7

C. An Approximation to the Concentration Profile 9

D. Calculating an Approximate Effectiveness Factor $\quad 12$

IV. TESTS OF THE THEORY $\quad 13$

A. Limiting Situations $\quad 13$

$\begin{array}{ll}\text { 1. Situation where no transport limitations exist } & 13\end{array}$

2. Situation where only mass transport limits reaction rate 13

B. Comparison of Results with Those from Rigorous Solution of
Equations Describing Intraparticle Heat and Mass Transport

V. DISCUSSION 23

A. Extensions to Other Situations

1. Possible elaborations 23

2. Extension to isothermal Langmuir-Hinshelwood reactions 23

3. Extending approach to nonisothermal Langmuir-Hinshelwood reactions 25

4. Extension to exothermic reactions 25

5. Extensions to non-spherical catalyst shapes $\quad 26$

B. Applicability of Work $\quad 26$

1. Simplicity of equations 26

2. Criteria for absence of heat and mass transfer effects $\quad 26$

3. Isothermal systems $\quad 26$

4. Nonisothermal systems $\quad 26$

$\begin{array}{lll}\text { VI. CONCLUSIONS } & 27\end{array}$

$\begin{array}{ll}\text { ACKNOWLEDGMENTS } & 28\end{array}$ 
APPENDIX A ANALYTIC INTEGRATION OF RHS OF EQ. (III-52)
A-1. Integration by Parts

page

A-1

A-2. Evaluating the Series Solution

A-1

A-2

APPENDIX B LISTING OF COMPUTER CODES USED IN THIS WORK

B-1

B-1. Basic Computer Code for Iterative Solution of Equation (IV-5) with Boundary Conditions Defined by Equations (IV-6)-(IV-7)

B-2. Basic Computer Code for Iterative Solution of Equations (IV-15) and (IV-16) with Boundary Conditions Defined by Equations (IV-6)-(IV-7) and (IV-17)-(IV-18)

\section{LIST OF TABLES}

Table 1. Isothermal Effectiveness Factor Values Predicted by Approximation Theory Compared with Rigorous Values

2. Endothermic Reaction Effectiveness Factors Predicted by Approximation Theory Compared with Rigorous Values-I $(\beta=-0.02, \varepsilon=20)$

3. Endothermic Reaction Effectiveness Factors Predicted by Approximation Theory Compared with Rigorous Values-II $(\beta=-0.02, \varepsilon=30)$

4. Endothermic Reaction Effectiveness Factors Predicted by Approximation Theory Compared with Rigorous Values-III $(\beta=-0.05, \varepsilon=20)$

5. Endothermic Reaction Effectiveness Factors Predicted by Approximation Theory Compared with Rigorous Values-IV $(\beta=-0.05, \varepsilon=30)$

6. Endothermic Reaction Effectiveness Factors Predicted by Approximation Theory Compared with Rigorous Values-V $(\beta=-0.1, \varepsilon=20)$

7. Endothermic Reaction Effectiveness Factors Predicted by Approximation Theory Compared with Rigorous Values-VI $(\beta=-0.1, \varepsilon=30)$

A-1 Basic Computer Code for Evaluation of Series Expansion of Analytic Solution to Integral on RHS of Eq. (III-52)

B-1 Basic Computer Code for Iterative Solution of Equation (IV-5) with Boundary Conditions Defined by Equations (IV-6)-(IV-7)

B-2 Basic Computer Code for Iterative Solution of Equations (IV-15) and (IV-16) with Boundary Conditions Defined by Equations (IV-6)-(IV-7) and (IV-17)-(IV-18) 


\section{NOMENCLATURE}

(Dimensions given below are typical dimensions. On occasion different units may be appropriate.)

Roman symbols
A Pre-exponential factor in Arrhenius expression for rate constant, $\left(\mathrm{m}^{3} / \mathrm{mol}\right)^{\mathrm{n}-1} \mathrm{~s}^{-1}$.
C Concentration, $\mathrm{mol} / \mathrm{m}^{3}$.
$D_{\text {eff }} \quad$ Effective diffusion coefficient within catalyst particle, $\mathrm{m}^{2} / \mathrm{s}$
$\mathrm{Da}_{\mathrm{IV}}$ Damköhler Group IV, dimensionless. For an $\mathrm{n}^{\text {th }}$-order reaction, $\mathrm{Da} \mathrm{a}_{\mathrm{IV}}=-\left(\Delta \mathrm{H}_{\mathrm{r}} \mathrm{L}^{2} \mathrm{Ae}^{-\varepsilon} \mathrm{C}_{\mathrm{A} 0}^{\mathrm{n}}\right) /\left(\lambda \mathrm{T}_{0}\right)$.
E The activation energy in Arrhenius expression for rate constants, $\mathrm{J} / \mathrm{mol}$.
$\mathrm{h} \quad$ Step size in Simpson's-rule numerical evaluation of an integral.
H Enthalpy, J/mol.
k The rate constant for a process. For this report, the dimensions are $\left(\mathrm{m}^{3}\right.$ of catalyst pellet) $/ \mathrm{mol}^{\mathrm{n}-1} \mathrm{~s}^{-1}$.
$\mathrm{k}$ One fewer than the number of terms in Simpson's-rule numerical evaluation of an integral.
k Index in series expansion of Appendix A's analytical evaluation of RHS of Eq. (III-52).
ln Natural logarithm.
L Characteristic length of a catalyst particle, $m$. In this report, the characteristic length is taken equal to the volume of the particle divided by its external surface area. For a spherical particle, $\mathrm{L}$ $=\mathrm{R} / 3$.
n Reaction order, dimensionless.
Q Rate of heat transfer, $\mathrm{J} / \mathrm{s}$
r Reaction rate, mols created $/(\mathrm{s})\left(\mathrm{m}^{3}\right.$ of catalyst particle).
$\mathrm{r}$ Distance from center of catalyst particle, $\mathrm{m}$.
R Gas constant, $8.314 \mathrm{~J} /(\mathrm{mol})(\mathrm{K})$ or $8.314 \times 10^{-6}(\mathrm{MPa})\left(\mathrm{m}^{3}\right) /(\mathrm{mol})(\mathrm{K})$.
$\mathrm{R} \quad$ Radius of spherical catalyst particle or effective radius of differently shaped particle, $\mathrm{m}$.
T Temperature, $\mathrm{K}$.
V Volume, $\mathrm{m}^{3}$.
$V_{p} \quad$ Gross volume of a single catalyst pellet, $\mathrm{m}^{3}$.

\section{Greek symbols}

$\alpha_{1} \quad$ Parameter in approximation to dimensionless temperature profile within catalyst particle, dimensionless. It is shown to have the relationship $\alpha_{1}=1+\left[\Delta H_{r}\left(-r_{A \exp }\right) R^{2} / 9 T_{0} \lambda\right]$.

$\alpha_{2}$ Parameter in approximation to dimensionless temperature profile within catalyst particle, dimensionless. Eliminated in preference to $\alpha_{1}$ through the relationship $\alpha_{2}=1-\alpha_{1}$ [Eq. (III-14)].

$\beta \quad$ The maximum intraphase adiabatic dimensionless temperature rise.

$\Delta \quad$ Change in a property upon completion of a reaction. 
$\mathrm{E} / \mathrm{RT}_{0}$, dimensionless. From the Maxwell-Boltzmann distribution of energies, it is the fraction of molecules with an energy greater than $\mathrm{E}$.

$\eta \quad$ Catalyst effectiveness factor, dimensionless.

$\theta \quad$ The dimensionless temperature defined by $\left(\mathrm{T}_{0} / \mathrm{T}\right)-1$.

$\theta 1$ The dimensionless temperature defined by $\mathrm{T} / \mathrm{T}_{0}$.

$\lambda \quad$ Effective heat transfer coefficient within catalyst particle, $J /(\mathrm{s})\left(\mathrm{m}^{2}\right)(\mathrm{K} / \mathrm{m})$, or $\mathrm{J} /(\mathrm{s})(\mathrm{m})(\mathrm{K})$.

$\xi \quad$ Dimensionless concentration of substance $A, C_{A} / C_{A 0}$.

$\pi \quad$ Ratio of a circle's circumference to its diameter, dimensionless.

$\rho \quad$ Dimensionless distance from particle center, $r / R$.

$\phi \quad$ Thiele modulus, dimensionless. For an $\mathrm{n}^{\text {th }}$-order reaction, $\phi=\mathrm{L}\left(\mathrm{kC}_{\mathrm{A} 0}{ }^{\mathrm{n}-1} / \mathrm{D}_{\mathrm{eff}}\right)^{1 / 2}$.

$\omega \quad$ Effectiveness factor multiplied by the square of the Thiele modulus, dimensionless. $\omega \equiv \eta \phi^{2}$. For an $n^{\text {th }}$-order reaction consuming a reactant $A, \eta \phi^{2}=-r_{A(\exp )} L^{2} / C_{A 0} D_{\text {eff }}$

\section{Subscripts}

A Of substance A.

exp Experimental value.

mass Indicates subscripted quantity is valid for a situation in which heat transfer effects are absent, and transfer only mass transfer influences the system.

$\mathrm{p} \quad$ Indicates subscripted quantity is that for the catalyst particle.

$r$ Refers to the change of the thermodynamic property during the chemical reaction in question.

0 Indicates that this is the value of the subscripted quantity at the exterior surface of a catalyst pellet or particle.

1 For $\mathrm{n}^{\text {th }}$-order reactions, this number indicates that the subscripted quantity is valid for a firstorder system; for Langmuir-Hinshelwood reactions, this number indicates that the subscripted quantity is valid for the value in the numerator of the rate expression.

1 For $\mathrm{n}^{\text {th }}$-order reactions, this number indicates that the subscripted quantity is valid for a secondorder system; for Langmuir-Hinshelwood reactions, this number indicates that the subscripted quantity is valid for the value in the denominator of the rate expression.

\section{ACRONYMS}

RHS Right-hand side of an equation or expression.

w.r.t. With respect to (an expression, equation, or parameter).

\section{GLOSSARY}

Catalyst effectiveness factor. Actual rate divided by rate that would occur if temperature of the entire catalyst were identical to the exterior surface temperature and if entire inner surface of the catalyst were exposed to the exterior concentrations of reactants and products.

Damköhler Group IV. In this report, it is energy that would be generated within a catalyst pellet if entire internal surface of the catalyst were exposed to the exterior temperature and concentrations, divided by an approximate heat-transfer rate within the pellet. It is dimensionless; $D a_{I V}=-\Delta H_{r} r_{0} L^{2} / \lambda T_{0}$. 
Rate Constant. In reaction-rate expressions, a proportionality factor times the component concentrations in the reactor raised to particular powers gives the reaction rate per unit time per unit volume or reaction rate per unit time per unit mass of catalyst. The proportionality factor is termed the rate constant. Ideally, it is independent of pressure or reactant concentration and depends only on temperature. In real situations, it often shows some dependence on pressure or reactant concentrations in addition to the temperature dependence. True units of the rate constant are [mols created $\left./(\mathrm{s})\left(\mathrm{m}^{3}\right)\right]\left(\mathrm{m}^{3} / \mathrm{mol}\right)^{\mathrm{n}}$ or [mols created $/(\mathrm{s})(\mathrm{kg})]\left(\mathrm{m}^{3} / \mathrm{mol}\right)^{\mathrm{n}}$; convention has canceled "mols created" with one of the mols in the denominator to yield $\left(\mathrm{m}^{3} / \mathrm{mol}\right)^{\mathrm{n}-1} \mathrm{~s}^{-1}$ or $\left(\mathrm{m}^{3}\right)^{\mathrm{n}} \mathrm{mol}^{\mathrm{n}-1}[(\mathrm{~kg})(\mathrm{s})]^{-1}$.

Syngas. Synthesis gas, a mixture of hydrogen and carbon monoxide. Depending on the final product made from the syngas, the ratio of hydrogen to carbon monoxide in the gas may vary from one to three.

Thiele modulus. A dimensionless group equal to the reaction rate in a pellet under extreme surfacerate limiting conditions divided by reaction rate in the pellet under extreme diffusion-rate-limiting conditions. 


\title{
SIMPLE RELATIONSHIPS FOR ESTIMATING INTRAPARTICLE TRANSPORT EFFECTS FOR CATALYTICALLY PROMOTED ENDOTHERMIC REACTIONS
}

\author{
Lee F. Brown \\ Engineering Science and Applications Division \\ Los Alamos National Laboratory, Los Alamos, NM 87544
}

\begin{abstract}
Relationships for estimating effectiveness factors for porous-solid-catalyzed fluid reactions can result from assuming approximations to temperature and concentration profiles. Approximations designed to simplify the outcome result in simple, explicit, analytic relationships for both isothermal and nonisothermal $\mathrm{n}^{\text {th }}$-order reaction systems. For isothermal systems, formulas developed predict effectiveness within $25 \%$ of the true isothermal effectiveness factors ( $\eta$ 's) over the range $0.1>\eta>0.99$. For isothermal or endothermic reaction systems with $\eta>0.65$, errors are less than $10 \%$. Even in the maximum-error region, estimates for endothermic systems are within a factor of two of those obtained by solution of the rigorous heat and mass transfer equations. For isothermal or endothermic systems with $\eta>0.95$, errors are less than $1 \%$. Thus the formulas can also serve diagnostic uses that confirm presence or absence of significant internal heat or mass transport effects in porous reacting systems. Extension of the approach to non- $n^{\text {th }}$-order reactions is possible; formulas are derived for simple isothermal and nonisothermal Langmuir-Hinshelwood reaction systems. Application of the work to exothermic reactions was not tested, but steeper gradients in such systems would tend to degrade accuracy of the relationships. The equations derived in this work are simpler and easier of application than any others proposed thus far.
\end{abstract}

\section{EXECUTIVE SUMMARY}

This report develops new and simpler equations for estimating effectiveness factors for porouscatalyst-promoted endothermic reactions. The relationships can also apply to fluid-solid reactions within porous materials. In addition, the formulas can serve diagnostic uses that detect presence of significant internal heat or mass transport effects in porous reacting systems.

Many previous investigators have studied this problem because of the tedious effort required to solve the rigorous mass and heat transfer equations involved. In the present report, approximations to temperature and concentration profiles enable derivation of equations for simple estimation of effectiveness factors. This work uses the following approximations for dimensionless temperatures and concentrations within a porous particle:

$$
\theta=\ln \left[\alpha_{1}+\left(1-\alpha_{1}\right) \rho^{3}\right] \text { (temperature) , } \quad \xi=\exp \left[-\omega\left(1-\rho^{3}\right)\right] \text { (concentration) . }
$$

Properties in common with those of rigorous profiles and mathematical simplicity of resulting formulas dictate the forms of these approximate profiles. In these approximations, $\alpha_{1}$ is a function of heat of reaction, external temperature, porous-particle radius, and experimental reaction rate. The parameter $\omega$ is a function of external concentrations, porous-particle radius, effective internal diffusivity of reactants, and 
experimental reaction rate. The dimensionless quantity $\rho$ is the ratio of distance from the particle center to particle radius.

Employing these approximations, the report derives explicit equations for estimating effectiveness factors for both isothermal and nonisothermal $n^{\text {th }}$-order reaction systems. For isothermal systems, the formula

$$
\eta \approx \frac{1}{\mathrm{n} \omega}\left(1-\mathrm{e}^{-\mathrm{n} \omega}\right)
$$

results. This relationship predicts effectiveness factors within $25 \%$ of true isothermal effectiveness factors over the range $0.1>\eta>0.99$. For effectiveness factors $\eta>0.65$, errors are less than $10 \%$. For effectiveness factors $\eta>0.95$, errors are less than $1 \%$.

For nonisothermal $\mathbf{n}^{\text {th }}$-order systems, the development derives the relationship

$$
\eta \approx \int_{0}^{1}\left[\alpha_{1}+\left(1-\alpha_{1}\right) y\right]^{-\varepsilon} \mathrm{e}^{-\mathrm{n} \omega(1-y)} \mathrm{dy}
$$

in which $\varepsilon$ is $\mathrm{E} / \mathrm{RT}_{0}$, the frequently-observed reaction activation energy term evaluated at the exterior temperature, and $\alpha_{1}$ is the parameter in the dimensionless temperature profile described above. The nonisothermal relationship requires numerical evaluation of the integral to obtain the approximate effectiveness factor. For effectiveness factors above 0.5, the three-term Simpson's rule,

$$
\eta \approx \frac{1}{6}\left[\left(\alpha_{1}\right)^{-\varepsilon} \mathrm{e}^{-\mathrm{n} \omega}+4\left(0.5+0.5 \alpha_{1}\right)^{-\varepsilon} \mathrm{e}^{-0.5 n \omega}+1\right]
$$

provides simply calculated estimates for endothermic reactions that are within $5 \%$ of values obtained using more elaborate numerical integrations. For lower effectiveness factors, more terms are needed in the Simpson's-rule integration to converge to the best value. Tests of the approximation compared its predictions for endothermic reactions with those calculated from solution of the appropriate rigorous mass and heat transfer equations. At effectiveness factors greater than 0.65 , errors are less than $10 \%$. At $\eta>0.95$, errors are less than $1 \%$. Accuracies are lower at lower effectiveness factors, presumably because steeper internal temperature and concentration gradients may cause the temperature and concentration-profile approximations to be less exact. Estimated factors for endothermic reactions are within a factor of two of the rigorous values even in regions of greatest deviations.

The approach is applicable to some non-integer-order reaction systems. Derivations demonstrate suitability of the concept in creating effectiveness-factor-approximation relationships for simple isothermal and nonisothermal Langmuir-Hinshelwood reaction systems. 
Rational approximations for effectiveness factors can be useful for diagnosis, i.e., to test for presence or absence of mass or heat effects in solid-catalyst-promoted reactions. The approximations developed here can serve this purpose easily. At values of $\eta \geq 0.95$, the approximations predict effectiveness factors within $1 \%$ of the rigorous values. As such, they can be used to judge presence or absence of mass or heat transport effects using any reasonable criterion deemed appropriate by an investigator's judgment.

While this work emphasizes endothermic reaction systems, its derivations make no assumption concerning endo- or exothermic natures of the reactions. Therefore they might have some use for estimating effectiveness factors of exothermic reactions as well as those of endothermic reactions. The study did not test accuracies of this approach for exothermic reactions, however. Typical temperature and concentration profiles within porous particles have steeper gradients in exothermic systems than in endothermic systems. As a result, estimates based on approximate profiles would probably give less accurate results for exothermic systems.

The derivations here assume spherical porous particles. Other geometric shapes used by various investigators include cylindrical and slab particles. Deriving equivalent equations for cylindrical particles is straightforward. Deriving the equations for a slab particle is not as simple, but might be possible.

The trend in developing rational relationships for approximating effectiveness factors has been toward ever more simple equations. The equations derived in this work are simpler and easier of application than any others proposed thus far.

\section{EFFECTIVENESS FACTORS}

\section{A. Calculation and Estimation of Effectiveness Factors}

A catalyst's effectiveness factor is the actual reaction rate divided by the rate that would occur if the entire inner surface were at the exterior surface temperature and exposed to exterior concentrations. This report develops new and simpler equations for estimating effectiveness factors for porous-catalystpromoted endothermic reactions. The relationships can also serve diagnostic purposes, for they can detect any significant internal heat or mass transfer effects in such systems. Additional work considers possible extensions to exothermic reactions.

Early investigators in this field began with analytic solutions to internally isothermal situations. In these cases, only mass transport affects the internal reaction. Internally isothermal systems are important, because thermal conductivities of catalyst particles are usually much higher than conductivities in external fluid boundary layers. Thus any temperature difference in a system may occur within the boundary layer, 
leaving the catalyst particle essentially isothermal. Carberry (1976) analyzes this situation, and gives criteria for judging whether a catalyst particle is or is not essentially isothermal.

Internally nonisothermal systems demand equations for both mass and heat transport within porous materials, and these require numerical solutions. Tinkler and Metzner (1961) and Weisz and Hicks (1962) were early in presenting such solutions. Aris (1975) reviews work with both isothermal and nonisothermal systems through 1973, and presents numerous insights into mathematical behavior of these systems.

The tedious nature of numerically solving simultaneous intraparticle mass and heat transport equations has led investigators to derive rational relationships that determine approximate values of effectiveness factors. This eases estimation of the factors. Many efforts consider only isothermal systems (e.g., Gottifredi et al. 1981a,b, Suenson et al. 1983, Haynes 1986, Gonzo et al. 1988, Yin and Li 1995a), but some treat nonisothermal systems as well (e.g., Kubota and Yamanaka 1969, Wedel and Luss 1980, Gonzo and Gottifredi 1982, 1983a.). Work in this area through 1982 is reviewed by Gonzo and Gottifredi (1983b). Since then, two trends appear. One is toward analyzing more complex systems (e.g., Gonzo et al. 1988, Goto and Ito 1990, Yin and Li 1995a,b), the other toward simpler relationships to reduce effort required for estimating effectiveness factors (e.g., Suenson et al. 1983, Haynes 1986). The present work continues the trend toward simpler approximate relationships.

\section{B. Diagnostic Criteria for Mass and Heat Transfer Limitations}

A second use of effectiveness-factor theory is determination whether intraparticle transport influences a fluid reaction catalyzed by a porous solid. This may be necessary for laboratory work in which the investigator is attempting to find the true kinetics of a catalytically promoted reaction.

Researchers have developed criteria for this purpose. (The criteria can also apply to a heterogeneous reaction between a fluid and a porous solid.) Weisz and Prater (1954) show that if the Thiele modulus squared times the effectiveness factor is significantly less than 1 in a first-order reaction system, the system is unaffected by intraparticle mass transport. Since the Thiele modulus squared times the effectiveness factor for an $n^{\text {th }}$-order reaction equals the quantity $\left|r_{\text {exp }}\right| L^{2} / D_{\text {eff }} C_{0}$, the criterion uses only a single rate measurement and easily measured or estimated quantities. Bischoff (1967) extends this criterion to include non-first-order reactions. Hudgins (1968) derives a criterion that, while less precise than the Weisz-PraterBischoff standard, is easier to apply for systems with non-integer reaction orders.

Another approach to discerning whether intraparticle transport effects exist uses relationships of the type mentioned above in Section A for approximating effectiveness factors. If an investigator judges that an effectiveness factor greater than 0.95 indicates no significant transport effects, then one of the rational relationships discussed above can determine whether the approximate effectiveness factor is greater or less 
than 0.95 . If the investigator's judgment decrees that the effectiveness factor must be greater than 0.99 to ensure absence of transport effects, the same relationship can be used with this criterion. These relationships tend to be more accurate at higher effectiveness factors, so they are usually reliable in this regard. They are also more precise than standards such as the Weisz-Prater-Bischoff or Hudgins criteria, that give only general guidelines as opposed to the exact measures possible with the rational approximations.

\section{Scope of This Work}

This work emphasizes catalytically promoted endothermic reactions. These reactions comprise a significant subset of chemical reactions with many of them producing large quantities of basic chemicals. Steam reforming of methane to form syngas $\left(\Delta \mathrm{H}_{\mathrm{r}(1000 \mathrm{~K})}=+226 \mathrm{~kJ} / \mathrm{mol}\right)$ and dehydrogenation of cyclohexane to form benzene $\left(\Delta \mathrm{H}_{\mathrm{r}(600 \mathrm{~K})}=+219 \mathrm{~kJ} / \mathrm{mol}\right)$ are but two examples of highly endothermic catalytically promoted reactions carried out on a mega-tonnage scale. The methanol-steam reaction $\left(\Delta \mathrm{H}_{\mathrm{r}(600 \mathrm{~K})}=+62 \mathrm{~kJ} / \mathrm{mol}\right)$, with its potential for creating a hydrogen-rich feed for fuel-cell-propelled automobiles, provided the original inspiration for this work.

The development carried out below first applies to endothermic $n^{\text {th }}$-order reactions within spherical catalyst pellets in which a significant decrease in temperature occurs. Relationships derived can give quantitatively adequate (within $10 \%$ ) estimates of catalyst effectiveness factors when the factors lie between 1 and 0.65 . They also give estimates within a factor of two of values rigorously obtained at all levels of effectiveness factor. Eliminating heat transport gives a simple relationship for isothermal systems that estimates effectiveness factors within $25 \%$ of rigorous values over the entire range of effectiveness factors tested. The discussion treats extensions of this work to Langmuir-Hinshelwood systems, to exothermic reaction systems, and to non-spherical geometries. General applications of the developments close the work. It is pointed out that the equations developed here for estimating effectiveness factors are accurate to within $1 \%$ at effectiveness factors above 0.95 . Thus the equations can determine precise effectiveness factors in the high range when an investigator wishes diagnostic criteria to ensure a lack of significant mass and heat transport effects.

\section{THEORETICAL DEVELOPMENT}

\section{A. Effectiveness Factor of a Spherical Catalyst Particle}

Because shapes of most real catalyst particles do not lend themselves to simple mathematical analysis, previous works in this area have considered three idealized particle shapes-slabs, cylinders, and spheres. This development considers a spherical particle, as this shape most closely resembles many actual 
catalysts available commercially. Extension of the approach to cylindrical models is direct. As mentioned earlier, the equations assume an $n^{\text {th }}$-order reaction. The development also assumes an Arrhenius temperature dependence of the rate constant.

The rate expression for a reaction consuming a substance $A$ that is $n^{\text {th }}$-order in $A$ is

$$
r_{\mathrm{A}}=-\mathrm{Ae}^{-\mathrm{E} / \mathrm{RT}} \mathrm{C}_{\mathrm{A}}^{\mathrm{n}}
$$

and the reaction rate of $\mathrm{A}$ within an entire catalyst particle is

$$
r_{A(p)}=-A \int_{0}^{V_{p}} e^{-E / R T} C_{A}^{n} d V
$$

Assuming a spherical particle, and changing the variable to distance from the particle center $r$, gives

$$
r_{A(p)}=-A \int_{0}^{R} e^{-E / R T} C_{A}^{n} \cdot 4 \pi r^{2} \cdot d r
$$

If there were no transport limitations, the total rate per particle would be

$$
r_{\mathrm{A}(\mathrm{p}) 0}=-\mathrm{Ae}^{-\mathrm{E} / \mathrm{RT}} \mathrm{C}_{\mathrm{A} 0}^{\mathrm{n}} \cdot \frac{4}{3} \pi \mathrm{R}^{3}
$$

The effectiveness factor $\eta$ of the catalyst particle (cf. Section II.A) would then be

$$
\eta=\frac{r_{A \exp }}{r_{A 0}}=\frac{r_{A(p)}}{r_{A(p) 0}}=\frac{3 \int_{0}^{R} e^{-E / R T} C_{A}^{n} r^{2} d r}{e^{-E / R T_{0}} C_{A 0}^{n} R^{3}}
$$

The dimensionless variables

$$
\xi \equiv \mathrm{C}_{\mathrm{A}} / \mathrm{C}_{\mathrm{A} 0}
$$

and

$$
\rho \equiv \mathbf{r} / \mathrm{R}
$$

can simplify Eq. (III-5). Dividing through by the denominator and substituting the two dimensionless variables into the equation give

$$
\eta=3 \int_{0}^{1} e^{-\frac{E}{R}\left(\frac{1}{T}-\frac{1}{T_{0}}\right)} \xi^{n} \rho^{2} d \rho
$$


Defining a dimensionless temperature as

$$
\theta \equiv \frac{T_{0}}{T}-1
$$

and substituting it into Eq. (III-8) give

$$
\eta=3 \int_{0}^{1} e^{-\frac{E \theta}{R T_{0}}} \xi^{n} \rho^{2} d \rho
$$

Let $\varepsilon \equiv \mathrm{E} / \mathrm{RT}_{0}$. This simplifies Eq. (III-10) to

$$
\eta=3 \int_{0}^{1} e^{-\varepsilon \theta} \xi^{n} \rho^{2} d \rho
$$

Both dimensionless temperature $\theta$ and dimensionless concentration $\xi$ are functions of the dimensionless radius $\rho$. Functional forms of these parameters are unknown a priori. To obtain useful results, past investigators have successfully approximated concentration profiles in pellets by simple functional forms, usually parabolas (e.g., Hudgins 1968, Stewart and Villadsen 1969, Brown 1972, Liaw et al. 1979, Rice 1982, Xiu et al. 1997). The next sections describe the functional forms chosen for this study's temperature and concentration profiles.

\section{B. An Approximation to the Temperature Profile}

The temperature profile within a spherical catalyst pellet must be symmetrical. A corollary to this has the derivative of temperature with respect to (w.r.t.) radius equal to zero at the pellet center. As mentioned above, past investigators have favored parabolas for profiles, but this study uses the natural logarithm of a cubic function:

$$
\theta=\ln \left(\alpha_{1}+\alpha_{2} \rho^{3}\right)
$$

Mathematical characteristics dictate this form. Use of this function results in a simple expression for the effectiveness factor possessing reasonable accuracy.

This approximation automatically satisfies the required symmetry condition at $\rho=0:\left.(d \theta / d \rho)\right|_{\rho=0}=0$. This form also implies that the natural logarithm of $\alpha_{1}$ is the value of $\theta$ at $\rho=0$. Looking at the 
definition of $\theta$ [Eq. (III-9)], its value at the particle's exterior surface $(\rho=1)$ is 0 . Substituting $\theta=0$ and $\rho=1$ into Eq. (III-12) gives

$$
0=\ln \left(\alpha_{1}+\alpha_{2}\right)
$$

This means that

$$
\alpha_{2}=1-\alpha_{1},
$$

and the approximation to the dimensionless temperature profile becomes

$$
\theta=\ln \left[\alpha_{1}+\left(1-\alpha_{1}\right) \rho^{3}\right]
$$

For a given overall reaction rate, the temperature gradient at the exterior surface of the pellet can provide the value of $\alpha_{1}$. Taking the derivative of $\theta$ w.r.t. $\rho$ in Eq. (III-15) and evaluating the derivative at $\rho=1$ give

$$
\left.\frac{d \theta}{d \rho}\right|_{\rho=1}=\frac{3\left(1-\alpha_{1}\right) \rho^{2}}{\alpha_{1}+\left(1-\alpha_{1}\right) \rho^{3}}=3\left(1-\alpha_{1}\right) .
$$

To obtain the gradient, the energy absorbed during reaction at steady state is equated to the energy entering the particle at the exterior surface. Relationships forming the balance are

$$
\mathrm{Q}_{0}=\Delta \mathrm{H}_{\mathrm{r}}\left(-\mathrm{r}_{\text {Aexp }}\right) \mathrm{V}_{\mathrm{p}}=\Delta \mathrm{H}_{\mathrm{r}}\left(-\mathrm{r}_{\text {Aexp }}\right)\left(\frac{4}{3} \pi \mathrm{R}^{3}\right)
$$

and

$$
\mathrm{Q}_{0}=\left.\lambda \cdot \mathrm{A}_{\mathrm{x}} \frac{\mathrm{dT}}{\mathrm{dr}}\right|_{\mathrm{r}=\mathrm{R}}=\left.\lambda \cdot 4 \pi \mathrm{R}^{2} \frac{\mathrm{dT}}{\mathrm{dr}}\right|_{\mathrm{r}=\mathrm{R}}
$$

Equating the right-hand sides (RHSs) of these two equations to each other gives

$$
\Delta \mathrm{H}_{\mathrm{r}}\left(-\mathrm{r}_{\text {Aexp }}\right)\left(\frac{4}{3} \pi \mathrm{R}^{3}\right)=\left.\lambda \cdot 4 \pi \mathrm{R}^{2} \frac{\mathrm{dT}}{\mathrm{dr}}\right|_{\mathrm{r}=\mathrm{R}}
$$

and

$$
\left.\frac{\mathrm{dT}}{\mathrm{dr}}\right|_{\mathrm{r}=\mathrm{R}}=\frac{\Delta \mathrm{H}_{\mathrm{r}}\left(-\mathrm{r}_{\mathrm{A} \exp }\right) \mathrm{R}}{3 \lambda}
$$

Solving Eq. (III-9) for $T$, taking the derivative w.r.t. $r$, then using $\rho=r / R$ give

$$
\frac{\mathrm{dT}}{\mathrm{dr}}=-\frac{\mathrm{T}_{0}}{(\theta+1)^{2} \mathrm{R}} \frac{\mathrm{d} \theta}{\mathrm{d} \rho}
$$


At $r=R(p=1), \theta=0$. Thus

$$
\left.\frac{\mathrm{dT}}{\mathrm{dr}}\right|_{\mathrm{r}=\mathrm{R}}=-\left.\frac{\mathrm{T}_{0}}{\mathrm{R}} \frac{\mathrm{d} \theta}{\mathrm{d} \rho}\right|_{\rho=1} .
$$

Substituting this into Eq. (III-20) and solving for $\left.(\mathrm{d} \theta / \mathrm{d} \rho)\right|_{\rho=1}$ yield

$$
\left.\frac{d \theta}{d \rho}\right|_{\rho=1}=\frac{\Delta H_{r} r_{A \exp } R^{2}}{3 T_{0} \lambda}
$$

Substituting the RHS of this equation for Eq. (III-16)'s gradient gives for $\alpha_{i}$,

$$
\alpha_{1}=1+\frac{\Delta \mathrm{H}_{\mathrm{r}}\left(-\mathrm{r}_{\mathrm{A} \exp }\right) \mathrm{R}^{2}}{9 \mathrm{~T}_{0} \lambda}
$$

The parameter $\alpha_{1}$ is a positive number greater than 1 for endothermic reactions, since $\theta$ is positive at all points within the particle $\left[\left(\mathrm{T}_{0} / \mathrm{T}\right)>1\right.$ everywhere]. Equation (III-24) reflects this.

\section{An Approximation to the Concentration Profile}

Just as the previous section derives a one-parameter approximation to the temperature profile, this section derives a one-parameter approximation to the concentration profile, $\xi=f(\rho), \quad 0 \leq \rho \leq 1$. The experimental information available that appears to be strongly related to internal concentration is the product appearing in the Weisz-Prater-Bischoff criterion, $\eta \phi^{2}$. For an $n^{\text {th }}$-order reaction consuming substance $A$ in a spherical catalyst particle,

$$
\eta \phi^{2}=\left(-\mathrm{r}_{\mathrm{Aexp}}\right) \mathrm{R}^{2} / 9 \mathrm{D}_{\mathrm{efr}} \mathrm{C}_{\mathrm{A} 0}
$$

Since this product is related to internal concentration, it is desirable that the parameter in the approximation be some direct function of $\eta \phi^{2}$.

The following delineates properties of the true profile; as such they are desirable properties of the approximate profile.

$$
\begin{aligned}
& \text { At } \rho=0:\left.\frac{d \xi}{d \rho}\right|_{\rho=0}=0 \quad \text { (the symmetry condition) } \\
& \text { At } \rho=1: \xi_{\rho=1}=1 \quad \text { (concentration at exterior surface) }
\end{aligned}
$$


To obtain the gradient at $\rho=1$, the moles of reactant consumed during the reaction at steady state are equated to moles of reactant entering the particle at the exterior surface. Relationships forming the balance are

$$
\mathrm{N}_{\mathrm{A} 0}=-\mathrm{r}_{\mathrm{Aexp}} \mathrm{V}_{\mathrm{p}}=-\mathrm{r}_{\mathrm{A} \exp } \cdot \frac{4}{3} \pi \mathrm{R}^{3}
$$

and

$$
\mathrm{N}_{\mathrm{A} 0}=\mathrm{D}_{\mathrm{eff}}-\left.4 \pi \mathrm{R}^{2} \cdot \frac{\mathrm{dC}}{\mathrm{dr}}\right|_{\mathrm{r}=\mathrm{R}}
$$

Equating RHSs of these two equations to each other gives

$$
-r_{\text {Aexp }} \cdot \frac{4}{3} \pi R^{3}=\left.D_{\text {eff }} 4 \pi R^{2} \cdot \frac{d C_{A}}{d r}\right|_{r=R}
$$

and

$$
\left.\frac{\mathrm{dC}_{\mathrm{A}}}{\mathrm{d} \mathbf{r}}\right|_{\mathrm{r}=\mathrm{R}}=\frac{-\mathrm{r}_{\mathrm{A} \exp } \mathrm{R}}{3 \mathrm{D}_{\mathrm{eff}}}
$$

Multiplying both sides of this equation by $\mathrm{R} \mathrm{C}_{\mathrm{A} 0}$ to convert it to dimensionless variables yields

$$
\left.\frac{d \xi}{d \rho}\right|_{\rho=1}=\frac{-r_{A \exp } R^{2}}{3 C_{A 0} D_{e f f}}
$$

Comparing the RHS of this equation with the value of $\eta \phi^{2}$ for a spherical catalyst particle given above [cf. Eq. III-25)] yields the limiting boundary condition:

$$
\text { At } \rho=1:\left.\frac{d \xi}{d \rho}\right|_{\rho=1}=3 \eta \phi^{2} \quad \text { (gradient at exterior surface of particle) }
$$

Additional limiting properties are:

$$
\begin{aligned}
& \text { As } \eta \phi^{2} \rightarrow 0: \xi \rightarrow 1,0 \leq \rho \leq 1 \text { (no transport effects) } \\
& \text { As } \eta \phi^{2} \rightarrow 0: \frac{d \xi}{d \rho} \rightarrow 0 \quad 0 \leq \rho \leq 1 \text { (no transport effects) } \\
& \text { As } \eta \phi^{2} \rightarrow \infty:\left.\frac{d \xi}{d \rho}\right|_{\rho=1} \rightarrow \infty
\end{aligned}
$$

$$
\text { (transport completely limiting; no reaction within particle) . }
$$




$$
\xi \geq 0,0 \leq \rho \leq 1 \text { (impossibility of negative concentrations). }
$$

As $\eta \phi^{2} \rightarrow \infty: \xi \rightarrow 0, \quad 0 \leq \rho<1$

(transport completely limiting; no reaction within particle)

After several trials, the function chosen for this study is

$$
\xi=\exp \left[-\eta \phi^{2}\left(1-\rho^{3}\right)\right] .
$$

To simplify notation, let $\omega \equiv \eta \phi^{2}$, so the dimensionless concentration approximation becomes

$$
\xi=\exp \left[-\omega\left(1-\rho^{3}\right)\right] \text {. }
$$

The derivative of this dimensionless concentration w.r.t. $\rho$ is

$$
\frac{d \xi}{d \rho}=3 \omega \rho^{2} e^{-\omega\left(1-\rho^{3}\right)}
$$

The limiting properties of this expression and its derivative are

$$
\text { At } \rho=0:\left.\frac{d \xi}{d \rho}\right|_{\rho=0}=0 \text {, }
$$

At $\rho=1: \xi_{\rho=1}=1$,

At $\rho=1:\left.\frac{d \xi}{d \rho}\right|_{\rho=1}=3 \omega$,

As $\omega \rightarrow 0: \xi \rightarrow 1,0 \leq \rho \leq 1$,

As $\omega \rightarrow 0: \frac{d \xi}{d \rho} \rightarrow 0 \quad 0 \leq \rho \leq 1$

As $\omega \rightarrow \infty:\left.\frac{d \xi}{d \rho}\right|_{\rho=1} \rightarrow \infty$,

$\xi \geq 0, \quad 0 \leq \rho \leq 1$,

and

$$
\text { as } \omega \rightarrow \infty: \xi \rightarrow 0, \quad 0 \leq \rho<1 \text {. }
$$

Thus the approximation, Eq. (III-40), and its derivative, Eq. (III-41), fulfill all eight desirable limiting properties of the ideal function and its derivative. 


\section{Calculating an Approximate Effectiveness Factor}

Substituting the temperature-profile approximation [Eq. (III-15)] and the concentration-profile approximation [Eq. (III-40)] into Eq. (III-11) yields

$$
\eta \approx 3 \int_{0}^{1} e^{-\varepsilon\left\{\ln \left[\alpha_{1}+\left(1-\alpha_{1}\right) \rho^{3}\right]\right\}} e^{-n \omega\left(1-\rho^{3}\right)} \rho^{2} d \rho
$$

Let $y \equiv \rho^{3}$, so $\mathrm{dy}=3 \rho^{2} \mathrm{~d} \rho$, and

$$
\eta \approx \int_{0}^{1} \mathrm{e}^{-\varepsilon\left\{\ln \left[\alpha_{1}+\left(1-\alpha_{1}\right) y\right]\right\}} \mathrm{e}^{-\mathrm{n} \omega(1-\mathrm{y})} \mathrm{dy}=\int_{0}^{1} \mathrm{e}^{\left\{\ln \left[\alpha_{1}+\left(1-\alpha_{1}\right) \mathrm{y}\right]\right\}(-\varepsilon)} \mathrm{e}^{-\mathrm{n} \omega(1-\mathrm{y})} \mathrm{dy},
$$

or

$$
\eta \approx \int_{0}^{1}\left[\alpha_{1}+\left(1-\alpha_{1}\right) y\right]^{-\varepsilon} e^{-n \omega(1-y)} d y
$$

Equation (III-52) is a simple expression giving the approximate effectiveness factor in terms of exterior surface temperature, activation energy, and two parameters $\alpha_{1}$ and $\omega$. Reaction characteristics and simple experimental measurements determine values of $\alpha_{1}$ [Eq. (III-24)] and $\omega\left[\equiv \eta \phi^{2}\right.$, Eq. (III-25)].

Though in principle the RHS of Eq. (III-52) can be integrated analytically (cf. Appendix A), the resulting series solution has a large number of terms that requires numerical summation. In addition, at high values of the Thiele modulus, the series diverges (cf. Appendix A). It is simpler and more widely applicable to evaluate the integral directly by numerical means. Numerical evaluation of the integral on the RHS of Eq. (III-52) is straightforward. For example, the 3-term Simpson's rule,

$$
\eta \approx \frac{h}{3}\left[f\left(y_{0}\right)+4 f\left(y_{1}\right)+f\left(y_{2}\right)\right]
$$

can obtain an approximate numerical evaluation of the integral. Choosing $h$ as 0.5 , and $y_{0}, y_{1}$, and $y_{2}$ as $0,0.5$, and 1.0 , respectively, give

$$
\eta \approx \frac{1}{6}\left[\left(\alpha_{1}\right)^{-\varepsilon} \mathrm{e}^{-\mathrm{n} \omega}+4\left(0.5+0.5 \alpha_{1}\right)^{-\varepsilon} \mathrm{e}^{-0.5 n \omega}+1\right]
$$

Simpson's rule for numerically evaluating integrals uses odd numbers of terms. With $\mathrm{k}$ an even number, the general $(k+1)$-term Simpson's rule is

$$
\eta \approx \frac{h}{3}\left[f\left(y_{0}\right)+4 f\left(y_{1}\right)+2 f\left(y_{2}\right)+4 f\left(y_{3}\right)+\ldots+2 f\left(y_{k-2}\right)+4 f\left(y_{k-1}\right)+f\left(y_{k}\right)\right]
$$


As $\mathrm{k}$ increases, this expression gives successively more accurate evaluations of the integral in Eq. (III-52). The general Simpson's rule evaluation of the integral in Eq. (III-52) is

$$
\begin{aligned}
\eta \approx & \frac{h}{3}\left\{\left(\alpha_{1}\right)^{-\varepsilon} \mathrm{e}^{-\mathrm{n} \omega}+4\left[\mathrm{~h}+(1-\mathrm{h}) \alpha_{1}\right]^{-\varepsilon} \mathrm{e}^{-(1-\mathrm{h}) n \omega}+2\left[2 \mathrm{~h}+(1-2 \mathrm{~h}) \alpha_{1}\right]^{-\varepsilon} \mathrm{e}^{-(1-2 h) n \omega}\right. \\
& \left.+4\left[3 \mathrm{~h}+(1-3 \mathrm{~h}) \alpha_{1}\right]^{-\varepsilon} \mathrm{e}^{-(1-3 \mathrm{~h}) n \omega}+\ldots+2\left[(\mathrm{k}-2) \mathrm{h}+2 \mathrm{~h} \alpha_{1}\right]^{-\varepsilon} \mathrm{e}^{-2 \mathrm{hn} \omega}+4\left[(\mathrm{k}-1) \mathrm{h}+\mathrm{h} \alpha_{1}\right]^{-\varepsilon} \mathrm{e}^{-\mathrm{hn} \omega}+1\right\},
\end{aligned}
$$

or

$$
\eta \approx \frac{1}{3 k}\left\{\begin{array}{l}
\alpha_{1}^{-\varepsilon} \mathrm{e}^{-\mathrm{n} \omega}+4\left[\frac{\mathrm{k}-1}{\mathrm{k}}+\left(1-\frac{\mathrm{k}-1}{\mathrm{k}}\right) \alpha_{1}\right]^{-\varepsilon} \mathrm{e}^{-\left(1-\frac{1}{\mathrm{k}}\right) \mathrm{n} \omega}+1 \\
+\sum_{\mathrm{i}=3,5,7, \text { etc. }}^{\mathrm{k}-3} 2\left[\frac{\mathrm{i}-1}{\mathrm{k}}+\left(1-\frac{\mathrm{i}-1}{\mathrm{k}}\right) \alpha_{1}\right]^{-\varepsilon} \mathrm{e}^{-\left(1-\frac{\mathrm{i}-1}{\mathrm{k}}\right) \mathrm{n} \omega}+4\left[\frac{\mathrm{i}}{\mathrm{k}}+\left(1-\frac{\mathrm{i}}{\mathrm{k}}\right) \alpha_{1}\right]^{-\varepsilon} \mathrm{e}^{-\left(1-\frac{\mathrm{i}}{\mathrm{k}}\right) \mathrm{n} \omega}
\end{array}\right\}
$$

\section{TESTS OF THE THEORY}

\section{A. Limiting Situations}

1. Situation where no transport limitations exist. A necessary condition for Eqs. (III-52)-(III-56) to be valid predictors of the effectiveness factor is that they give an effectiveness factor of 1 when no transport limitations exist. In this situation, both temperature and concentration profiles are flat, $\alpha_{1}=1$, and $\omega=0$. When $\alpha_{1}=1$ and $\omega=0$, Eq. (III-52) becomes simply

$\eta_{\text {no transport limitations }}=\int_{0}^{1}\left[\alpha_{1}+\left(1-\alpha_{1}\right) y\right]^{-\varepsilon} \mathrm{e}^{-\mathrm{n} \omega(1-\mathrm{y})} \mathrm{dy}=\int_{0}^{1}[1+(1-1) \mathrm{y}]^{-\varepsilon} \mathrm{e}^{-(\mathrm{n})(0)(1-\mathrm{y})} \mathrm{dy}=\int_{0}^{1} \mathrm{dy}=1$

This first necessary condition is thus satisfied. The parameter $\alpha_{1}$ may approach one when there is a low heat of reaction, a low reaction rate, a high thermal conductivity, or any combination of these. Whatever the cause, the temperature profile is flat when no transport limitations exist. Similarly, an approximate zero value of $\omega$ may result from a low reaction rate or a high effective diffusion coefficient. Either way, the concentration profile is also flat when no transport limitations exist.

2. Situation where only mass transport limits reaction rate. As mentioned earlier, thermal conductivities of catalyst particles are usually significantly higher than conductivities of fluids composing the boundary layers. For this reason, the principal temperature drop in either an endothermic or exothermic 
system may occur within the boundary layer, leaving the catalyst particle essentially isothermal. Under these circumstances, $\theta \approx 0$ throughout the catalyst particle and Eq. (III-11) becomes

$$
\eta_{\text {mass transfer }}=\frac{r_{A(p)}}{r_{A(p) 0}}=3 \int_{0}^{1} \xi^{n} \rho^{2} d \rho
$$

Substituting the concentration-profile approximation [Eq. (III-40)] into this equation and employing $y \equiv \rho^{3}$ result in

$$
\eta_{\text {mass transfer }} \approx \int_{0}^{1} \mathrm{e}^{-\mathrm{n} \omega(1-\mathrm{y})} \mathrm{dy}=\frac{1}{\mathrm{n} \omega}\left(1-\mathrm{e}^{-\mathrm{n} \omega}\right)
$$

Since the integral in Eq. (IV-3) can be integrated analytically, there is no need for numerical evaluation to estimate the effectiveness factor when heat transport effects are negligible. This approximation should be valid when only intraparticle mass transport influences the system.

As $\theta \rightarrow 0$ throughout the particle, the dimensionless temperature at the particle center $\alpha_{1} \rightarrow 1$. This leads to a second approach to obtaining Eq. (IV-3). Substituting $\alpha_{1}=1$ into Eq. (III-52) gives the limiting approximation Eq. (IV-3).

The accuracy of Eq. (IV-3)'s approximation can be evaluated. For $n^{\text {th }}$-order systems, the rigorous mass transport equation describing behavior of component $\mathrm{A}$ within the catalyst particle is

$$
0=D_{\text {eff }}\left(\frac{d^{2} C_{A}}{d r^{2}}+\frac{2}{r} \frac{d C_{A}}{d r}\right)-k C_{A}^{n} .
$$

Converting this equation to non-dimensional form uses the same dimensionless concentration $\left(\xi \equiv \mathrm{C}_{\mathrm{A}} / \mathrm{C}_{\mathrm{A} 0}\right)$ and radius $(\rho \equiv r / R)$ as before, and uses the Thiele modulus for an $n^{\text {th }}$-order reaction $\left[\phi=L\left(k C_{A 0}^{n-1} / D_{\text {eff }}\right)^{1 / 2}\right]$. The characteristic length L suggested by Aris (1965), Bischoff (1965) and Petersen (1965) for spherical catalyst particles is $L=R / 3$. Using these relationships results in

$$
0=\frac{d^{2} \xi}{d \rho^{2}}+\frac{2}{\rho} \frac{d \xi}{d \rho}-\frac{\phi^{2}}{9} \xi^{n} .
$$

The two boundary conditions needed to solve this equation are

$$
\begin{array}{lll}
\xi=1 & \text { at } & \rho=1 \\
\text { and } & \text { at } & \rho=0 .
\end{array}
$$


If a situation specifies $\phi$ or terms composing this dimensionless group, Eq. (IV-5) can be solved to give the dimensionless concentration $\xi$ as a function of dimensionless radius for $0 \leq p \leq 1$. The effectiveness factor can then be evaluated from Eq. (IV-2):

$$
\eta_{\text {mass transfer }}=3 \int_{0}^{1} \xi^{n} \rho^{2} d \rho
$$

When the reaction being considered is first order, Eq. (IV-5) is linear and investigators have obtained analytic solutions. For spherical particles, Eq. (IV-8) expresses $\eta$ in terms of $\phi$ :

$$
\eta=\frac{1}{\phi} \frac{3 \phi \operatorname{coth} 3 \phi-1}{3 \phi} \text { (Aris 1969). }
$$

It follows directly that

$$
\omega=\eta \phi^{2}=\phi \operatorname{coth} 3 \phi-\frac{1}{3}
$$

Thus, if $\phi$ is specified, Eq. (IV-8) can calculate the rigorous $\eta$ and Eq. (IV-9) can calculate $\omega$. Equation (IV-3) can then calculate the $\eta$ predicted by the approximation theory. The $\eta$ 's predicted by the approximation theory can be compared with rigorous $\eta$ 's calculated from Eq. (IV-8), testing the theory.

For non-first-order systems, the mass balance is nonlinear, so computation of the rigorous effectiveness factor usually requires numerical procedures. Nevertheless, an analogous procedure can still test the approximation theory. If a situation specifies $\phi$ or terms composing this dimensionless group, Eq. (IV-5) can be solved numerically to give the dimensionless concentration $\xi$ as a function of dimensionless radius for $0 \leq p \leq 1$. The effectiveness factor can then be evaluated from Eq. (IV-2). Given $\phi$ and the rigorous effectiveness factor, $\omega$ follows. Equation (IV-3) then gives the approximation-theory estimate of the effectiveness factor. Appendix B gives the text of the code used for solving Eq. (IV-5) and calculating effectiveness factors for a second-order system.

Using these approaches, calculations obtained rigorous and approximate effectiveness factors for first and second-order systems for Thiele moduli in the range $0.1 \leq \phi \leq 10$. Table 1 presents results of these tests. Qualitatively, the table shows that the approximation theory can tell immediately whether intraparticle mass transport is influencing reaction rate. If the approximation theory predicts the isothermal effectiveness factor to be significantly less than 1 , the rigorous isothermal effectiveness factor is also going to be significantly less than 1 . 
Table 1

\section{ISOTHERMAL EFFECTIVENESS-FACTOR VALUES PREDICTED BY APPROXIMATION THEORY COMPARED WITH RIGOROUS VALUES}

$\begin{array}{rcccc}\phi & \begin{array}{c}\eta_{1} \\ \text { (rigorous) }\end{array} & \begin{array}{c}\eta_{1} \\ \text { (approx. } \\ \text { theory) }\end{array} & \begin{array}{c}\eta_{2} \\ \text { (rigorous) }\end{array} & \begin{array}{c}\eta_{2} \\ \text { (approx. } \\ \text { theory) }\end{array} \\ 0.1 & 0.994 & 0.995 & 0.988 & 0.990 \\ 0.2 & 0.977 & 0.981 & 0.956 & 0.963 \\ 0.3 & 0.950 & 0.958 & 0.909 & 0.922 \\ 0.4 & 0.916 & 0.930 & 0.86 & 0.87 \\ 0.6 & 0.83 & 0.86 & 0.75 & 0.77 \\ 0.8 & 0.75 & 0.79 & 0.65 & 0.68 \\ 1.0 & 0.67 & 0.73 & 0.57 & 0.60 \\ 2.0 & 0.42 & 0.49 & 0.34 & 0.34 \\ 4.0 & 0.23 & 0.27 & 0.19 & 0.17 \\ 6.0 & 0.16 & 0.18 & 0.13 & 0.11 \\ 8.0 & 0.12 & 0.13 & 0.10 & 0.078 \\ 10.0 & 0.097 & 0.10 & 0.079 & 0.061\end{array}$

Quantitatively, the table shows that the approximating Eq. (IV-3) predicts effectiveness factors within $25 \%$ of true effectiveness factors over the entire range of Thiele moduli. For effectiveness factors $\eta>0.65$, errors are less than $10 \%$. For effectiveness factors $\eta>0.95$, errors are less than $1 \%$.

The last observation is a reflection of the convergence of both the rigorous and approximation calculations of $\eta_{\text {mass transfer }}$ to a value of 1 as $\omega$ approaches 0 . Thus the approximation theory should become more accurate as $\omega \rightarrow 1$.

At the other end of the Thiele modulus spectrum, both rigorous and approximation calculations converge to a value of $\eta_{\text {mass transfer }}$ of 0 as $\omega$ approaches $\infty$. The Table 1 numbers at the highest values of the Thiele modulus also reflect this.

The limiting condition says that if heat transfer effects are eliminated from Eq. (III-52), the result should approximate rigorous calculations of isothermal effectiveness factors. Table 1 shows that this limiting condition is satisfied.

The isothermal approximation, Eq. (IV-3), gives estimates that in some cases are not as accurate as those given by approximations of Suenson et al. (1983) and Haynes (1986). Nevertheless, Eq. (IV-3) is simpler than relationships proposed by these investigators. 


\section{B. Comparison of Results with Those from Rigorous Solution of Equations Describing Intraparticle Heat and Mass Transport}

This section gives a thorough test of Eqs. (III-52)-(III-56). It compares effectiveness factors they predict for typical endothermic situations with factors obtained from the same situations' rigorous heat and mass transport equations. An $n^{\text {th }}$-order nonisothermal reaction requires that the steady-state mass balance for substance A, Eq. (IV-5), be modified to include the rate constant's temperature dependence. The result is

$$
0=D_{\text {eff }}\left(\frac{d^{2} C_{A}}{d r^{2}}+\frac{2}{r} \frac{d C_{A}}{d r}\right)-A e^{-E / R T} C_{A}^{n}
$$

The steady-state energy balance is

$$
0=\lambda\left(\frac{d^{2} T}{d r^{2}}+\frac{2}{r} \frac{d T}{d r}\right)-\Delta H_{r} A e^{-E / R T} C_{A}^{n}
$$

Converting these equations to non-dimensional form uses the same dimensionless concentration and radius as before, but employs a different dimensionless temperature. Let

$$
\theta 1 \equiv \frac{T}{T_{0}}
$$

The Thiele modulus is evaluated at the exterior surface temperature:

$$
\phi \equiv L \sqrt{\frac{A e^{-E / R T_{0}} C_{A 0}^{n-1}}{D_{e f f}}}
$$

Introducing the Damköhler Group IV (Catchpole and Fulford 1966) as a second parameter gives

$$
\mathrm{Da}_{I V} \equiv \frac{-\Delta \mathrm{H}_{\mathrm{r}} \mathrm{L}^{2} A \mathrm{e}^{-\mathrm{E} / \mathrm{RT}_{0}} \mathrm{C}_{\mathrm{A} 0}^{\mathrm{n}}}{\lambda \mathrm{T}_{0}}=\frac{-\Delta \mathrm{H}_{\mathrm{r}} \mathrm{L}^{2} \mathrm{Ae}^{-\varepsilon} \mathrm{C}_{\mathrm{A} 0}^{\mathrm{n}}}{\lambda \mathrm{T}_{0}}
$$

The characteristic length $L=R / 3$ is again employed. Using the Thiele modulus and Damköhler group defined above as parameters and reduced variables defined earlier, Eqs. (IV-10) and (IV-11) become

$$
0=\frac{d^{2} \xi}{d \rho^{2}}+\frac{2}{\rho} \frac{d \xi}{d \rho}-\frac{\phi^{2}}{9} e^{-\varepsilon\left(\frac{1}{\theta 1}-1\right)} \xi^{n}
$$

and

$$
0=\frac{d^{2} \theta 1}{d \rho^{2}}+\frac{2}{\rho} \frac{d \theta 1}{d \rho}-\frac{D a_{I V}}{9} e^{-\varepsilon\left(\frac{1}{\theta 1}-1\right)} \xi^{n}
$$


The four boundary conditions required to solve these equations are Eqs. (IV-6) and (IV-7) plus

\begin{tabular}{|c|c|c|c|}
\hline & $\theta 1=1$ & at & $\rho=1$ \\
\hline nd & $\frac{d \theta 1}{d \rho}=0$ & at & $\rho=0$ \\
\hline
\end{tabular}

As for the isothermal case, the system possesses a split-boundary-value nature.

The dimensionless parameter used to characterize non-isothermality of intraparticle-reaction systems is the maximum intraphase adiabatic dimensionless temperature rise, i.e.,

$$
\beta \equiv \frac{-\Delta H_{r} D_{e f f} C_{A 0}}{\lambda T_{0}}=\frac{D a_{I V}}{\phi^{2}}
$$

For an endothermic reaction $\left(\Delta \mathrm{H}_{\mathrm{r}}>0\right), \beta$ is negative. As an aside, combining Eqs. (III-24), (III-25) and $($ IV-19) gives

$$
\beta=\frac{1-\alpha_{1}}{\omega}
$$

in the approximate criterion theory. Values of $\beta$ in the range of $-0.1<\beta<-0.02$ characterize significantly endothermic reactions. For example, in a typical methanol-steam system with a reaction possessing a $\Delta \mathrm{H}_{\mathrm{r}}$ of $+60 \mathrm{~kJ} / \mathrm{mol}$, a $\mathrm{D}_{\text {eff }}$ of $10^{-5} \mathrm{~m}^{2} / \mathrm{s}$, a bulk methanol concentration of $7 \mathrm{~mol} / \mathrm{m}^{3}$, a catalyst $\lambda$ of $0.4 \mathrm{~W} /(\mathrm{m})(\mathrm{K})$ and a bulk temperature of $550 \mathrm{~K}$, there exists a $\beta$ of -0.02 . For reactions with greater changes of enthalpy, such as dehydrogenation of cyclohexane to produce benzene $\left(\Delta \mathrm{H}_{\mathrm{r}(600 \mathrm{~K})}=+219 \mathrm{~kJ} / \mathrm{mol}\right)$, the value of $\beta$ can approach -0.1 .

If a situation specifies both $\phi$ and $\beta$ or terms composing these dimensionless numbers, Eq. (IV-20) specifies Damköhler group IV. All parameters and boundary conditions in Eqs. (IV-16) and (IV-17) are

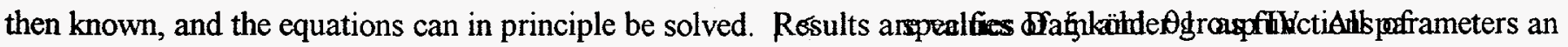
dimensionless radius for $0 \leq \rho \leq 1$. Using these values, the effectiveness factor can be evaluated from Eq. (III-10). The equation is changed to the following form because of the change in dimensionlesstemperature definition:

$$
\eta=3 \int_{0}^{1} e^{-\varepsilon\left(\frac{1}{\theta 1}-1\right)} \xi^{n} \rho^{2} \mathrm{~d} \rho
$$

Numerical solution of Eqs. (IV-15), (IV-16), and (IV-21) for a set of conditions gives the rigorous effectiveness factor for those conditions. Codes for solving the equations and calculating these effectiveness factors were written. Graphical values presented by Tinkler and Metzner (1961) and Weisz and Hicks (1962) checked some of the codes' calculations. Appendix B of this report gives a text copy of 
the code used for solving the three equations. The code solves the equations for first-order systems, but modifications for other $\mathrm{n}^{\text {th }}$-order systems follow without complication.

Estimation of effectiveness factor by the proposed relationship requires parameters $\alpha_{1}$ and $\omega$. If $\phi$ is specified and $\eta$ calculated from the rigorous approach, $\omega$ results from $\omega=\eta \phi^{2}$. Knowing $\beta$ and $\omega$, Eq. (IV-20) gives $\alpha_{1}$. Knowing $\alpha_{1}$ and $\omega$ enables calculation of estimated effectiveness factors via Eq. (III-54) or (III-56) for given values of $\phi, E / \mathrm{RT}_{0}$, and $\beta$.

Using first and second-order reactions, two sets of the factor $E / \mathrm{RT}_{0}$, and three sets of the maximum adiabatic temperature rise $\beta$, Tables 2 through 7 compare rigorous effectiveness factors and those estimated by the approximate theory for different values of the Thiele modulus. Values of $\beta$ chosen, $-0.02,-0.05$, and -0.1 , are shown above to encompass the range of significantly endothermic reactions. The values of $\mathrm{E} / \mathrm{RT}_{0}$ chosen, 20 and 30 , are also representative of catalytically promoted reactions. Again using the methanol-steam system as an example, with an activation energy of $85 \mathrm{~kJ} / \mathrm{mol}$ and a typical reaction temperature of $525 \mathrm{~K}, \mathrm{E} / \mathrm{RT}_{0}=19.5$ for this system.

Tables 2 and 3 present comparisons of the rigorous and estimated effectiveness factors for systems with a $\beta$ of -0.02 and $E / R_{0} T^{\prime} s(\varepsilon ' s)$ of 20 and 30 . Thiele moduli range from 0.1 to 10 . For this $\beta$, the estimated effectiveness factor shows a maximum deviation from the rigorous value of $22 \%$ at the highest Thiele modulus and higher $\varepsilon$. At lower Thiele-modulus values, the deviation decreases until it is less than $1 \%$ at the lowest Thiele moduli. Thus agreement between estimation-theory and rigorous values is excellent over the entire range of Thiele moduli for moderately endothermic reactions.

Equation (III-54), the 3-term Simpson's rule approximation to the integral in Eq. (III-52), is a particularly simple formula for estimating effectiveness factors for endothermic reactions. Table 2 shows that this formula does not begin to deviate significantly from higher-order Simpson's-rule approximations until the estimated effectiveness factor drops below 0.25 . Thus for the higher effectiveness factors, the 3 term Simpson's rule is entirely adequate for estimating effectiveness factors, and is much simpler than any other relationship proposed to date. The 3-term Simpson's rule approximation converges to an estimated effectiveness factor of 0.17 at low Thiele-modulus values. As a result, use of the 3-term Simpson's rule should not be considered if the estimated effectiveness factor is below about 0.25 .

For reactions with a higher endothermicity, with a $\beta$ of -0.05 , Tables 4 and 5 show the maximum deviation of the estimated effectiveness factor from the rigorous value to be $36 \%$. This deviation again occurs at the lowest Thiele modulus and higher $\varepsilon$. As before, deviations decrease with decreasing Thiele modulus and increasing effectiveness factor, until the deviations become less than $1 \%$ at the lowest Thiele moduli. Again the 3-term Simpson's-rule approximation gives excellent agreement with the higher rigorous 
effectiveness factors, though this time the lower limit for good agreement is about 0.4 . Below this value of effectiveness factor, higher-order Simpson's-rule computations are needed.

For the highest endothermicity $(\beta=-0.1)$, Tables 6 and 7 show that $43 \%$ is the maximum deviation of the estimated from the rigorous factor. As in the previous two tables, the percentage deviation decreases with decreasing Thiele modulus and increasing effectiveness factor. At the lowest Thiele moduli, the estimated effectiveness factors once again lie within $1 \%$ of the rigorous values.

Table 1, discussed earlier, presents the outcomes occurring when $\beta=0$, and these results must be included in any overview of the accuracy of the approximations. Over the entire range of variables investigated, the estimated values are within $10 \%$ of the rigorous numbers for effectiveness factors greater than 0.65. At lower effectiveness factors, the steeper gradients may cause the temperature and concentration-profile approximations to be less accurate, with a resulting loss in accuracy of the estimated factors. Nevertheless, estimated effectivenesses are within a factor of two of the rigorous values even in the regions of greatest deviations.

Table 2

\section{ENDOTHERMIC REACTION EFFECTIVENESS FACTORS PREDICTED BY APPROXIMATION THEORY COMPARED WITH RIGOROUS VALUES - I}

\begin{tabular}{|c|c|c|c|c|c|c|c|}
\hline & & & $\beta=-0.02$, & & & & \\
\hline & 1st-Orde & - Reaction & & & 2nd-Ord & r Reaction & \\
\hline$\eta($ exact $)$ & $\begin{array}{c}\eta \\
\text { (3-term } \\
\text { approx) }\end{array}$ & $\begin{array}{l}\eta \text { (2-fig. } \\
\text { accuracy } \\
\text { approx) }\end{array}$ & $\begin{array}{l}k^{*} \text { req'd } \\
\text { for } 2 \text {-fig. } \\
\text { accuracy }\end{array}$ & $\begin{array}{c}\eta \\
\text { (exact) }\end{array}$ & $\begin{array}{c}\eta \\
\text { (3-term } \\
\text { approx) }\end{array}$ & $\begin{array}{c}\eta \text { (2-fig. } \\
\text { accuracy } \\
\text { approx) }\end{array}$ & $\begin{array}{l}k^{*} \text { req'd } \\
\text { for } 2 \text {-fig. } \\
\text { accuracy }\end{array}$ \\
\hline 0.993 & 0.993 & 0.99 & 2 & 0.988 & 0.988 & 0.99 & 2 \\
\hline 0.970 & 0.973 & 0.97 & 2 & 0.950 & 0.956 & 0.96 & 2 \\
\hline 0.935 & 0.943 & 0.94 & 2 & 0.900 & 0.909 & 0.91 & 2 \\
\hline 0.89 & 0.91 & 0.91 & 2 & 0.84 & 0.86 & 0.86 & 2 \\
\hline 0.80 & 0.82 & 0.82 & 2 & 0.72 & 0.74 & 0.74 & 2 \\
\hline 0.71 & 0.74 & 0.74 & 2 & 0.63 & 0.64 & 0.64 & 2 \\
\hline 0.63 & 0.66 & 0.66 & 2 & 0.55 & 0.56 & 0.56 & 2 \\
\hline 0.39 & 0.41 & 0.41 & 2 & 0.33 & 0.31 & 0.31 & 2 \\
\hline 0.22 & 0.23 & 0.20 & 4 & 0.18 & 0.19 & 0.14 & 8 \\
\hline 0.15 & 0.18 & 0.13 & 6 & 0.13 & 0.17 & 0.093 & 10 \\
\hline 0.11 & 0.17 & 0.10 & 6 & 0.096 & 1 & 0.068 & 14 \\
\hline 0.093 & $\downarrow$ & 0.077 & 14 & 0.078 & $\downarrow$ & 0.054 & 14 \\
\hline
\end{tabular}

* This is the $k$ in the Simpson's-rule evaluation of the integral in Eq. (52). Simpson's rule has k+l terms. 
Table 3

\section{ENDOTHERMIC REACTION EFFECTIVENESS FACTORS PREDICTED BY APPROXIMATION THEORY COMPARED WITH RIGOROUS VALUES-II}

$$
\beta=-0.02, \varepsilon=30
$$

1st-Order Reaction

$\begin{array}{llccc}\phi \quad \eta(\text { exact }) & \eta & \eta \text { (2-fig. } & k^{*} \text { req'd } \\ & \text { (3-term } & \text { accuracy } & \text { for } 2 \text {-fig. } \\ & \text { approx) } & \text { approx) } & \text { accuracy }\end{array}$

$\begin{array}{rllll}0.1 & 0.992 & 0.992 & 0.99 & 2 \\ 0.2 & 0.966 & 0.970 & 0.97 & 2 \\ 0.3 & 0.927 & 0.936 & 0.94 & 2 \\ 0.4 & 0.88 & 0.90 & 0.90 & 2 \\ 0.6 & 0.78 & 0.81 & 0.81 & 2 \\ 0.8 & 0.69 & 0.72 & 0.72 & 2 \\ 1.0 & 0.61 & 0.64 & 0.64 & 2 \\ 2.0 & 0.38 & 0.38 & 0.38 & 2 \\ 4.0 & 0.21 & 0.21 & 0.19 & 4 \\ 6.0 & 0.15 & 0.18 & 0.12 & 6 \\ 8.0 & 0.11 & 0.17 & 0.089 & 14 \\ 10.0 & 0.090 & \downarrow & 0.070 & 12\end{array}$

2nd-Order Reaction

$\begin{array}{cccc}\eta & \eta & \eta \text { (2-fig. } & k^{*} \text { req'd } \\ \text { (exact) } & \text { (3-term } & \text { accuracy } & \text { for } 2 \text {-fig. } \\ & \text { approx) } & \text { approx) } & \text { accuracy }\end{array}$

0.986
0.946
0.890
0.83
0.71
0.61
0.54
0.32
0.18
0.12
0.094
0.076

0.987

$0.99 \quad 2$

0.952

0.95

0.90

0.85

0.73

0.85

0.73

0.63

0.63

0.54

0.54

0.30

0.14

0.18

0.17

0.088

0.064

0.051

2
2
2
2
2
2
2
2
4
10
18
16

Table 4

\section{ENDOTHERMIC REACTION EFFECTIVENESS FACTORS PREDICTED BY} APPROXIMATION THEORY COMPARED WITH RIGOROUS VALUES-III

$$
\beta=-0.05, \varepsilon=20
$$

1st-Order Reaction

$\phi \quad \eta($ exact $)$

$\begin{array}{ccc}\eta & \eta \text { (2-fig. } & k^{*} \text { req'd } \\ \text { (3-term } & \text { accuracy } & \text { for 2-fig. } \\ \text { approx) } & \text { approx) } & \text { accuracy }\end{array}$

$\begin{array}{rllll}0.1 & 0.990 & 0.990 & 0.99 & 2 \\ 0.2 & 0.958 & 0.963 & 0.96 & 2 \\ 0.3 & 0.912 & 0.922 & 0.92 & 2 \\ 0.4 & 0.86 & 0.87 & 0.87 & 2 \\ 0.6 & 0.75 & 0.77 & 0.77 & 2 \\ 0.8 & 0.66 & 0.68 & 0.68 & 2 \\ 1.0 & 0.58 & 0.59 & 0.59 & 2 \\ 2.0 & 0.36 & 0.34 & 0.33 & 4 \\ 4.0 & 0.20 & 0.20 & 0.16 & 4 \\ 6.0 & 0.14 & 0.17 & 0.10 & 6 \\ 8.0 & 0.105 & \downarrow & 0.075 & 18 \\ 10.0 & 0.085 & \downarrow & 0.060 & 14\end{array}$

\section{2nd-Order Reaction}

$\begin{array}{cccc}\eta & \eta & \eta \text { (2-fig. } & k^{*} \text { req'd } \\ \text { (exact) } & \text { (3-term } & \text { accuracy } & \text { for } 2 \text {-fig. } \\ & \text { approx) } & \text { approx) } & \text { accuracy }\end{array}$

$\begin{array}{llll}0.984 & 0.985 & 0.99 & 2 \\ 0.938 & 0.946 & 0.94 & 2 \\ 0.88 & 0.89 & 0.89 & 2 \\ 0.81 & 0.83 & 0.83 & 2 \\ 0.69 & 0.71 & 0.70 & 4 \\ 0.59 & 0.60 & 0.60 & 2 \\ 0.52 & 0.51 & 0.51 & 2 \\ 0.31 & 0.28 & 0.26 & 6 \\ 0.17 & 0.18 & 0.12 & 8 \\ 0.12 & 0.17 & 0.080 & 12 \\ 0.090 & \downarrow & 0.059 & 14 \\ 0.073 & \downarrow & 0.046 & 20\end{array}$

* This is the k in the Simpson's-rule evaluation of the integral in Eq. (52). Simpson's rule has k+1 terms. 
Table 5

\section{ENDOTHERMIC REACTION EFFECTIVENESS FACTORS PREDICTED BY} APPROXIMATION THEORY COMPARED WITH RIGOROUS VALUES-IV

$$
\beta=-0.05, \varepsilon=30
$$

1st-Order Reaction

\begin{tabular}{|c|c|c|}
\hline$\eta($ exact $)$ & $\begin{array}{c}\eta \\
\text { (3-term } \\
\text { approx) }\end{array}$ & $\begin{array}{l}\eta \text { (2-fig. } \\
\text { accuracy } \\
\text { approx) }\end{array}$ \\
\hline
\end{tabular}

$\begin{array}{rllllllll}0.1 & 0.987 & 0.988 & 0.99 & 2 & 0.982 & 0.983 & 0.98 & 2 \\ 0.2 & 0.945 & 0.954 & 0.95 & 2 & 0.929 & 0.938 & 0.94 & 2 \\ 0.3 & 0.894 & 0.906 & 0.91 & 2 & 0.86 & 0.88 & 0.88 & 2 \\ 0.4 & 0.84 & 0.85 & 0.85 & 2 & 0.79 & 0.81 & 0.81 & 2 \\ 0.6 & 0.72 & 0.74 & 0.74 & 2 & 0.67 & 0.68 & 0.68 & 2 \\ 0.8 & 0.63 & 0.63 & 0.63 & 2 & 0.57 & 0.57 & 0.57 & 2 \\ 1.0 & 0.55 & 0.55 & 0.54 & 4 & 0.49 & 0.48 & 0.48 & 2 \\ 2.0 & 0.33 & 0.30 & 0.29 & 4 & 0.29 & 0.26 & 0.24 & 4 \\ 4.0 & 0.18 & 0.19 & 0.14 & 6 & 0.16 & 0.17 & 0.11 & 6 \\ 6.0 & 0.13 & 0.17 & 0.087 & 10 & 0.11 & & 0.072 & 14 \\ 8.0 & 0.098 & \downarrow & 0.065 & 12 & 0.085 & & 0.053 & 16 \\ 10.0 & 0.080 & \downarrow & 0.051 & 16 & 0.069 & \downarrow & 0.042 & 18\end{array}$

Table 6
2nd-Order Reaction

$\begin{array}{cccc}\eta & \eta & \eta \text { (2-fig. } & k^{*} \text { req'd } \\ \text { (exact) } & \text { (3-term } & \text { accuracy } & \text { for } 2 \text {-fig. } \\ & \text { approx) } & \text { approx) } & \text { accuracy }\end{array}$

$10.0 \quad 0.080$ ENDOTHERMIC REACTION EFFECTIVENESS FACTORS PREDICTED BY
APPROXIMATION THEORY COMPARED WITH RIGOROUS VALUES-V

$\beta=-0.1, \varepsilon=20$

1st-Order Reaction

$\phi \quad \eta($ exact $)$

\section{2nd-Order Reaction}

$\begin{array}{cccc}\eta & \eta & \eta \text { (2-fig. } & k^{*} \text { req'd } \\ \text { (exact) } & \text { (3-term } & \text { accuracy } & \text { for } 2 \text {-fig. } \\ & \text { approx) } & \text { approx) } & \text { accuracy }\end{array}$

$\begin{array}{rllll}0.1 & 0.984 & 0.985 & 0.99 & 2 \\ 0.2 & 0.938 & 0.946 & 0.95 & 2 \\ 0.3 & 0.88 & 0.89 & 0.89 & 2 \\ 0.4 & 0.81 & 0.83 & 0.83 & 2 \\ 0.6 & 0.69 & 0.71 & 0.71 & 2 \\ 0.8 & 0.60 & 0.60 & 0.60 & 2 \\ 1.0 & 0.52 & 0.51 & 0.51 & 2 \\ 2.0 & 0.31 & 0.28 & 0.27 & 4 \\ 4.0 & 0.17 & 0.18 & 0.13 & 6 \\ 6.0 & 0.12 & 0.17 & 0.079 & 12 \\ 8.0 & 0.092 & \downarrow & 0.058 & 16 \\ 10.0 & 0.075 & \downarrow & 0.046 & 16\end{array}$

$\begin{array}{llll}0.979 & 0.981 & 0.98 & 2 \\ 0.921 & 0.930 & 0.93 & 2 \\ 0.85 & 0.86 & 0.86 & 2 \\ 0.77 & 0.79 & 0.79 & 2 \\ 0.65 & 0.65 & 0.65 & 2 \\ 0.55 & 0.54 & 0.54 & 2 \\ 0.47 & 0.45 & 0.45 & 2 \\ 0.28 & 0.24 & 0.22 & 4 \\ 0.15 & 0.17 & 0.10 & 8 \\ 0.11 & \mid & 0.067 & 12 \\ 0.081 & \mid & 0.049 & 18 \\ 0.066 & \downarrow & 0.038 & 46\end{array}$

* This is the $\mathrm{k}$ in the Simpson's-rule evaluation of the integral in Eq. (52). Simpson's rule has k+1 terms. 
Table 7

\section{ENDOTHERMIC REACTION EFFECTIVENESS FACTORS PREDICTED BY} APPROXIMATION THEORY COMPARED WITH RIGOROUS VALUES - VI

\begin{tabular}{|c|c|c|c|c|c|c|c|c|}
\hline & & & & $\beta=-0.1, \varepsilon$ & & & & \\
\hline & & 1st-Orde & Reaction & & & 2nd-Ord & r Reaction & \\
\hline$\phi$ & $\eta$ (exact) & $\begin{array}{c}\eta \\
\text { (3-term } \\
\text { approx) }\end{array}$ & $\begin{array}{l}\eta(2-\text { fig. } \\
\text { accuracy } \\
\text { approx })\end{array}$ & $\begin{array}{l}k^{*} \text { req'd } \\
\text { for } 2 \text {-fig. } \\
\text { accuracy }\end{array}$ & $\begin{array}{c}\eta \\
\text { (exact) }\end{array}$ & $\begin{array}{c}\eta \\
\text { (3-term } \\
\text { approx) }\end{array}$ & $\begin{array}{l}\eta(2-f i g . \\
\text { accuracy } \\
\text { approx) }\end{array}$ & $\begin{array}{l}k^{*} \text { req'd } \\
\text { for } 2 \text {-fig. } \\
\text { accuracy }\end{array}$ \\
\hline 0.1 & 0.979 & 0.981 & 0.98 & 2 & 0.973 & 0.976 & 0.98 & 2 \\
\hline 0.2 & 0.921 & 0.930 & 0.93 & 2 & 0.905 & 0.915 & 0.91 & 2 \\
\hline 0.3 & 0.85 & 0.86 & 0.86 & 2 & 0.82 & 0.84 & 0.84 & 2 \\
\hline 0.4 & 0.76 & 0.79 & 0.79 & 2 & 0.74 & 0.75 & 0.75 & 2 \\
\hline 0.6 & 0.65 & 0.65 & 0.65 & 2 & 0.61 & 0.61 & 0.61 & 2 \\
\hline 0.8 & 0.55 & 0.54 & 0.54 & 2 & 0.51 & 0.49 & 0.49 & 2 \\
\hline 1.0 & 0.48 & 0.45 & 0.45 & 2 & 0.44 & 0.41 & 0.40 & 4 \\
\hline 2.0 & 0.28 & 0.24 & 0.22 & 6 & 0.26 & 0.22 & 0.19 & 8 \\
\hline 4.0 & 0.16 & 0.17 & 0.10 & 6 & 0.14 & 0.17 & 0.090 & 12 \\
\hline 6.0 & 0.108 & & 0.066 & 12 & 0.097 & & $0 . .058$ & 16 \\
\hline 8.0 & 0.082 & & 0.049 & 16 & 0.075 & & 0.042 & 20 \\
\hline 10.0 & 0.067 & $\downarrow$ & 0.038 & 22 & 0.060 & $\downarrow$ & 0.033 & 54 \\
\hline
\end{tabular}

* This is the $\mathrm{k}$ in the Simpson's rule-evaluation of the integral in Eq. (52). Simpson's rule has $\mathrm{k}+1$ terms.

\section{DISCUSSION}

\section{A. Extensions to Other Situations}

1. Possible elaborations. Equations derived thus far in this work assume an $n^{\text {th }}$-order reaction within a porous spherical particle. Tests examined only endothermic reactions. Possible elaborations of the approach are therefore to non- ${ }^{\text {th }}$-order reactions and reactions within slab and cylindrical porous particles, and consideration of applications to exothermic reaction systems.. The following sections consider these extensions.

2. Extension to isothermal Langmuir-Hinshelwood reactions. As noted earlier, the isothermal situation can simplify the mathematical development significantly. The simplest Langmuir-Hinshelwood rate is

$$
r=\frac{-A_{1} e^{-E_{1} / R T} C_{A}}{1+A_{2} e^{-E_{2} / R T} C_{A}}
$$


For a reaction with this rate expression within an isothermal particle $\left(T=T_{0}\right.$ throughout the particle), Eq. (III-5) becomes

$$
\eta_{\text {mass transfer }}=\frac{3 A_{1} \mathrm{e}^{-\mathrm{E}_{1} / \mathrm{RT}_{0}} \int_{0}^{\mathrm{R}}\left(\frac{\mathrm{C}_{\mathrm{A}}}{1+\mathrm{A}_{2} \mathrm{e}^{-\mathrm{E}_{2} / \mathrm{RT}_{0}} \mathrm{C}_{\mathrm{A}}}\right) \mathrm{r}^{2} \mathrm{dr}}{\mathrm{A}_{1} \mathrm{e}^{-\mathrm{E}_{1} / \mathrm{RT}_{0}}\left(\frac{\mathrm{C}_{\mathrm{A} 0}}{1+\mathrm{A}_{2} \mathrm{e}^{-\mathrm{E}_{2} / \mathrm{RT}_{0}} \mathrm{C}_{\mathrm{A} 0}}\right) \mathrm{R}^{3}} .
$$

Canceling where appropriate, dividing both numerator and denominator by $C_{A 0} /\left(1+A_{2} e^{-E_{2} / R_{0}} C_{A 0}\right)$, and substituting dimensionless parameters $\xi$ and $\rho$ for their equivalents give

$$
\eta_{\text {mass transfer }}=3\left(1+\mathrm{A}_{2} \mathrm{e}^{-\mathrm{E}_{2} / \mathrm{RT}_{0}} \mathrm{C}_{\mathrm{A} 0}\right) \int_{0}^{1} \frac{\xi}{1+\mathrm{A}_{2} \mathrm{e}^{-E_{2} / R T_{0}} \mathrm{C}_{\mathrm{A} 0} \xi} \rho^{2} \mathrm{~d} \rho
$$

Letting $\gamma \equiv \mathrm{A}_{2} \mathrm{e}^{-\mathrm{E}_{2} / \mathrm{RT}_{0}} \mathrm{C}_{\mathrm{A} 0}$ and substituting this into Eq. (V-3) give

$$
\eta_{\text {mass transfer }}=3(1+\gamma) \int_{0}^{1} \frac{\xi}{1+\gamma \xi} \rho^{2} \mathrm{~d} \rho
$$

Again letting $y \equiv \rho^{3}$ (cf. Eq. III-51) and $\xi \approx \mathrm{e}^{-\omega(1-y)}$, then substituting these into Eq. (V-4), gives

$$
\eta_{\text {mass transfer }} \approx(1+\gamma) \int_{0}^{1} \frac{\mathrm{e}^{-\omega(1-y)}}{1+\gamma \mathrm{e}^{-\omega(1-y)}} d y=(1+\gamma) \int_{0}^{1} \frac{1}{\mathrm{e}^{\omega(1-y)}+\gamma} d y
$$

This can be integrated to give

$$
\eta_{\text {mass transfer }} \approx \frac{1}{\gamma}\left[\frac{1}{\omega} \ln \left(\frac{\gamma+\mathrm{e}^{\omega}}{\gamma+1}\right)-1\right]
$$

If all parameters in the rate expression are known, then all parameters in Eq. (V-6) are either known or in principle obtainable by simple experiments. Analogous derivations for approximate isothermal effectiveness factors may be possible for reactions possessing more complex Langmuir-Hinshelwood rate expressions. 
3. Extending approach to nonisothermal Langmuir-Hinshelwood reactions. For the same simple Langmuir-Hinshelwood reaction, but with a significant temperature gradient within the porous particle, the $T_{0}$ 's in the numerator of Eq. $(V-2)$ become $T$ :

$$
\eta=\frac{3 A_{1} \int_{0}^{R} e^{-E_{1} / R T}\left(\frac{C_{A}}{1+A_{2} e^{-E_{2} / R T} C_{A}}\right) r^{2} d r}{A_{1} e^{-E_{1} / R_{0}}\left(\frac{C_{A 0}}{1+A_{2} e^{-E_{2} / R_{0}} C_{A 0}}\right) R^{3}}
$$

Canceling where appropriate, dividing both numerator and denominator by $C_{A 0} d\left(1+A_{2} e^{-E_{2} / R T_{0}} C_{A 0}\right)$, and substituting dimensionless parameters $\theta, \xi$ and $\rho$ for their equivalents give

$$
\eta=3\left(1+\mathrm{A}_{2} \mathrm{e}^{-\mathrm{E}_{2} / \mathrm{RT}_{0}} \mathrm{C}_{\mathrm{A} 0}\right) \int_{0}^{1} \frac{\mathrm{e}^{-\mathrm{E}_{1} \theta / R \mathrm{~T}_{0} \xi}}{1+\mathrm{A}_{2} \mathrm{e}^{-\mathrm{E}_{2} / \mathrm{RT}_{0}} \mathrm{C}_{\mathrm{A} 0} \mathrm{e}^{-\mathrm{E}_{2} \theta / \mathrm{RT} \mathrm{T}_{0} \xi}} \rho^{2} \mathrm{~d} \rho
$$

Substituting parameters $\mathrm{y} \equiv \rho^{3}, \gamma \equiv \mathrm{A}_{2} \mathrm{e}^{-\mathrm{E}_{2} / \mathrm{RT}_{0}} \mathrm{C}_{\mathrm{A} 0}, \varepsilon_{1} \equiv \frac{\mathrm{E}_{1}}{\mathrm{RT}_{0}}$, and $\varepsilon_{2} \equiv \frac{\mathrm{E}_{2}}{\mathrm{RT}_{0}}$, plus approximations $\xi \approx \mathrm{e}^{-\omega(1-y)}$ and $\theta \approx \ln \left[\alpha_{1}+\left(1-\alpha_{1}\right) \rho^{3}\right]$, results in

$$
\eta=(1+\gamma) \int_{0}^{1} \frac{\left[\alpha_{1}+\left(1-\alpha_{1}\right) y\right]^{-\varepsilon_{1}} e^{-\omega(1-y)}}{1+\gamma\left[\alpha_{1}+\left(1-\alpha_{1}\right) y\right]^{-\varepsilon_{2}} e^{-\omega(1-y)}} d y
$$

If kinetic parameters in the rate expression and exterior concentrations are available, $\alpha_{1}, \gamma, \varepsilon_{1}$ and $\varepsilon_{2}$ can be calculated. Knowing these values enables straightforward numerical integration of Eq. (V-9). Thus approximation of effectiveness factors is possible with this approach for at least some types of Langmuir-Hinshelwood reactions.

4. Extension to exothermic reactions. The derivation of Eq. (III-52) makes no assumption concerning the endo- or exothermic nature of the reaction. Therefore in principle it should be usable for estimating effectiveness factors of exothermic reactions as well as endothermic reactions. Accuracy of this approach for exothermic reactions has not yet been determined, however. Typical temperature and concentration profiles within porous particles have steeper gradients in exothermic systems than in endothermic systems, and these might lead to less accurate results. This effect was noted earlier when discussing the less accurate results for endothermic systems at high Thiele moduli. Thus results for exothermic systems have a potential for being less satisfactory than those for endothermic systems.

For particular regions in the $\eta-\phi$ space of some exothermic reactions, there are multiple solutions of the system of equations (cf. Tinkler and Metzner 1961, Weisz and Hicks 1962). Equation (III-52) will not 
give multiple solutions, so it clearly is not applicable to this region of the $\eta-\phi$ space of particular exothermic reactions.

5. Extensions to non-spherical catalyst shapes. Deriving equivalent equations for cylindrical particles is straightforward. Wherever a $\rho^{3}$ appears in the derivation, a $\rho^{2}$ is substituted, i.e., in approximations to temperature and concentration profiles. Then the variable y would equal $\rho^{2}$ instead of $\rho^{3}$, and integration is direct.

Deriving an equivalent equation for a slab particle is not as simple. Further manipulation of variables and parameters would be necessary, because a linear approximation to the temperature profile is not realistic.

\section{B. Applicability of Work}

1. Simplicity of equations. As mentioned earlier, the trend in developing rational relationships for approximating effectiveness factors has been toward ever more simple equations. Relationships developed in this work continue this trend, being simpler and easier of application than any others proposed thus far.

2. Criteria for absence of heat and mass transfer effects. Sections I.B and I.C mention that rational approximations for effectiveness factors can implement criteria for demonstrating absence of mass or heat effects in solid-catalyst-promoted reactions. Approximations developed in this work can serve this purpose easily. At values of $\eta \geq 0.95$, the approximations predict effectiveness factors within $1 \%$ of rigorous values. As such, they can be used to judge absence of mass or heat transport effects using any reasonable criterion deemed appropriate by judgment of the investigator (e.g., $\eta \geq 0.98$ ).

3. Isothermal systems. For $n^{\text {th }}$-order isothermal systems, the quantity $\eta \phi^{2}$ is readily obtainable. In addition, investigators present plots of $\eta$ as a function of $\eta \phi^{2}$ for first-order systems (e.g., Weisz and Prater 1954, Carberry 1976). Thus for first-order isothermal systems, the value of $\eta$ may be calculated rigorously as described in Section IV.A.2, it may be read off a plot of $\eta$ as a function of $\eta \phi^{2}$, or it may be estimated by using Eq. (IV-3) in the present work.. The rigorous calculation is annoying and tedious, and the graphs cannot be read to give precise values. Depending on the accuracy that an investigator desires, Eq. (IV-3) probably gives reasonable values and is the simplest approach available. If a situation requires more accuracy, the approximations of Suenson et al. (1983) or Haynes (1986) are available but require more effort.

4. Nonisothermal systems. While analytical solutions exist for some isothermal systems, determining rigorous effectiveness factors for any nonisothermal system still requires solution of the simultaneous split-boundary value ODEs analogous to Eqs. (IV-15) and (IV-16). These require iterative calculations, and evaluation of expressions such as Eq. (III-52) and (V-9) is much simpler. This is the 
inspiration behind the series of rational approximations derived in this area's previous work, and the idea behind the present effort.

This work's approach gives its most accurate predictions at small values of $\beta$ and smaller values of $\varepsilon$. This means that it performs best for lower heats of reaction, smaller particles, higher exterior surface temperatures, higher solid thermal conductivities, and lower reaction activation energies. Nevertheless, at its poorest this approach estimates effectiveness factors within a factor of two, which often is sufficient. At effectiveness factors greater than 0.65 , this approach gives quantitatively adequate (within $10 \%$ ) estimates for almost all purposes.

\section{CONCLUSIONS}

A relationship for estimating effectiveness factors of spherical particles in $\mathbf{n}^{\text {th }}$-order isothermal systems is

$$
\eta_{\text {mass transfer }} \approx \frac{1}{n \omega}\left(1-\mathrm{e}^{-\mathrm{n} \omega}\right)
$$

in which $\omega$ equals the effectiveness factor times the Thiele modulus squared $\left(\eta \phi^{2}\right)$. Effectiveness factors calculated using this equation lie within $25 \%$ of factors calculated by solving the rigorous mass transfer equations for these systems over almost the entire possible range of effectiveness factors. Analogous expressions can be derived for at least some Langmuir-Hinshelwood isothermal systems.

A relationship for estimating effectiveness factors of spherical particles in $\mathrm{n}^{\text {th }}$-order nonisothermal systems is

$$
\eta \approx \int_{0}^{1}\left[\alpha_{1}+\left(1-\alpha_{1}\right) y\right]^{-\varepsilon} \mathrm{e}^{-n \omega(1-y)} \mathrm{dy}
$$

in which $\omega$ has the same meaning as above, and $\alpha_{1}=1-\left(\mathrm{r}_{\mathrm{Aexp}} \Delta \mathrm{H}_{\mathrm{r}} \mathrm{R}^{2} / 9 \mathrm{~T}_{0} \lambda\right)$. Numerical evaluation of the integral is straightforward. For endothermic reactions, this equation gives a quantitatively adequate estimate (within $10 \%$ ) of a catalyst effectiveness factor when that factor is greater than 0.65 . Estimates of lower effectiveness factors exhibit greater errors, though the estimates are always within a factor of two of rigorous values. Analogous relationships can be derived for at least some Langmuir-Hinshelwood nonisothermal systems. Accuracy of Eq. (III-52) for exothermic reactions is as yet undetermined, but is likely to be poorer than that for endothermic reactions.

The 3-term Simpson's rule approximation to the integral in Eq. (III-52), 


$$
\eta \approx \frac{1}{6}\left[\left(\alpha_{1}\right)^{-\varepsilon} \mathrm{e}^{-\mathrm{n} \omega}+4\left(0.5+0.5 \alpha_{1}\right)^{-\varepsilon} \mathrm{e}^{-0.5 \mathrm{n} \omega}+1\right],
$$

is a particularly simple relationship that gives 2-figure accuracy for the numerical integration at estimated effectiveness factors above 0.5 .

The relationships can be used in a diagnostic fashion to determine whether mass or heat transport influences an endothermic fluid-porous solid reaction. At effectiveness factors greater than 0.95 , they estimate factors within $1 \%$ of those calculated by solution of rigorous mass and heat transport equations, so their use as diagnostic equations gives precise answers. The relationships for exothermic systems have potential for this use, but their accuracy is as yet undetermined.

These relationships are mathematically the simplest yet derived for these purposes.

\section{ACKNOWLEDGMENTS}

Professor Eric Nuttall of the University of New Mexico's Chemical and Nuclear Engineering Department was very helpful in indicating how to solve some of the differential-equation systems in this work. Conversations with Professor Henry Haynes of the University of Wyoming and Professors Abhaya Datye and Lee Perry of the University of New Mexico lent insights. This work was carried out under the auspices of the U.S. Department of Energy. 


\section{APPENDIX A}

\section{ANALYTIC INTEGRATION OF RHS OF EQUATION (III-52)}

\section{A-1. Integration by Parts}

The object is to evaluate analytically the integral

$$
\int_{0}^{1}\left[\alpha_{1}+\left(1-\alpha_{1}\right) y\right]^{-\varepsilon} e^{-n \omega(1-y)} d y
$$

The equality

$$
\int_{0}^{1}\left[\alpha_{1}+\left(1-\alpha_{1}\right) y\right]^{-\varepsilon} e^{-n \omega(1-y)} d y=e^{-n \omega} \int_{0}^{1}\left[\alpha_{1}+\left(1-\alpha_{1}\right) y\right]^{-\varepsilon} e^{+n \omega y} d y
$$

reduces the problem to evaluating analytically the integral

$$
\int_{0}^{1}\left[\alpha_{1}+\left(1-\alpha_{1}\right) y\right]^{-\varepsilon} e^{n \omega y} d y
$$

Integration by parts,

$$
\int_{a}^{b} u d v=\left.u v\right|_{a} ^{b}-\int_{a}^{b} v d u
$$

applied to Eq. (A-3), with

$u=\left[\alpha_{1}+\left(1-\alpha_{1}\right) y\right]^{-\varepsilon}, \quad d u=(-\varepsilon)\left(1-\alpha_{1}\right)\left[\alpha_{1}+\left(1-\alpha_{1}\right) y\right]^{-\varepsilon-1} d y, \quad d v=n \omega e^{n \omega y} d y, \quad v=e^{n \omega y}$,

gives

$$
\begin{aligned}
& \frac{1}{\mathrm{n} \omega} \int_{0}^{1}\left[\alpha_{1}+\left(1-\alpha_{1}\right) y\right]^{-\varepsilon} n \omega \mathrm{e}^{\mathrm{n} \omega \mathrm{y}} \mathrm{dy} \\
& \quad=\frac{1}{\mathrm{n} \omega}\left\{\left.\left[\alpha_{1}+\left(1-\alpha_{1}\right) \mathrm{y}\right]^{-\varepsilon} \mathrm{e}^{\mathrm{n} \omega \mathrm{y}}\right|_{0} ^{1}+\varepsilon\left(1-\alpha_{1}\right) \int_{0}^{1}\left[\alpha_{1}+\left(1-\alpha_{1}\right) y\right]^{-\varepsilon-1} \mathrm{e}^{\mathrm{n} \omega \mathrm{y}} \mathrm{dy}\right\} \\
& \quad=\frac{1}{\mathrm{n} \omega}\left\{\mathrm{e}^{\mathrm{n} \omega}-\alpha_{1}^{-\varepsilon}+\varepsilon\left(1-\alpha_{1}\right) \int_{0}^{1}\left[\alpha_{1}+\left(1-\alpha_{1}\right) \mathrm{y}\right]^{-(\varepsilon+1)} \mathrm{e}^{\mathrm{n} \omega \mathrm{y}} \mathrm{dy}\right\}
\end{aligned}
$$


Following the same procedure to evaluate the integral on the RHS of Eq. (A-7), mutatis mutandis, results in

$$
\int_{0}^{1}\left[\alpha_{1}+\left(1-\alpha_{1}\right) y\right]^{-(\varepsilon+1)} e^{n \omega y} d y=\frac{1}{n \omega}\left\{e^{n \omega}-\alpha_{1}^{-(\varepsilon+1)}+(\varepsilon+1)\left(1-\alpha_{1}\right) \int_{0}^{1}\left[\alpha_{1}+\left(1-\alpha_{1}\right) y\right]^{-(\varepsilon+2)} e^{n \omega y} d y\right\}
$$

Continuously evaluating the integrals leads to a series with ever-increasing negative exponents. This means that the series will eventually converge. The resulting series is

$$
\begin{aligned}
& \int_{0}^{1}\left[\alpha_{1}+\left(1-\alpha_{1}\right) y\right]^{-\varepsilon} \mathrm{e}^{-\mathrm{n} \omega(1-\mathrm{y})} \mathrm{dy} \\
& \quad=\mathrm{e}^{-\mathrm{n} \omega}\left\{\left(\frac{1}{\mathrm{n} \omega}\right)\left(\mathrm{e}^{\mathrm{n} \omega}-\alpha_{1}^{-\varepsilon}\right)+\sum_{\mathrm{k}=2}^{\infty}\left(\frac{1}{\mathrm{n} \omega}\right)^{\mathrm{k}}\left[\mathrm{e}^{\mathrm{n} \omega}-\alpha_{1}{ }^{-(\varepsilon+\mathrm{k}-1)}\right]\left(1-\alpha_{1}\right)^{\mathrm{k}-1}[(\varepsilon)(\varepsilon+1) \ldots(\varepsilon+\mathrm{k}-2)]\right\} \\
& \quad=\left(\frac{1}{\mathrm{n} \omega}\right)\left(1-\alpha_{1}{ }^{-\varepsilon} \mathrm{e}^{-\mathrm{n} \omega}\right)+\sum_{\mathrm{k}=2}^{\infty}\left(\frac{1}{\mathrm{n} \omega}\right)^{\mathrm{k}}\left(1-\alpha_{1}{ }^{-(\varepsilon+\mathrm{k}-1)} \mathrm{e}^{-\mathrm{n} \omega}\right)\left(1-\alpha_{1}\right)^{\mathrm{k}-1}[(\varepsilon)(\varepsilon+1) \ldots(\varepsilon+\mathrm{k}-2)] .
\end{aligned}
$$

\section{A-2 Evaluating the Series Solution}

Equation (A-10) requires numerical evaluation. The code listed in Table A-1 evaluates Eq. (A-10) for the conditions $\phi=1, \eta_{\text {rigorous }}=0.63, \beta=-0.02, \varepsilon=20$, and $n=1$. Using these parameters, the results are

\begin{tabular}{rc}
\multicolumn{1}{c}{ term } & sum \\
+0.9292 & +0.9292 \\
-0.3750 & +0.5542 \\
+0.1588 & +0.7131 \\
-0.0705 & +0.6426 \\
+0.0327 & +0.6753 \\
-0.0158 & +0.6595 \\
+0.0080 & +0.6674 \\
-0.0042 & +0.6633 \\
+0.0023 & +0.6655 \\
-0.0013 & +0.6643 \\
+0.0007 & +0.6650 \\
-0.0005 & +0.6646 \\
+0.0003 & +0.6648 \\
-0.0002 & +0.6647 \\
+0.0001 & +0.6648 \\
-0.0001 & +0.6647 \\
+0.0001 & +0.6648
\end{tabular}




$$
\begin{array}{rr}
-0.0000 & +0.6647 \\
+0.0000 & +0.6647 \\
-0.0000 & +0.6647 \\
+0.0000 & +0.6647
\end{array}
$$

The terms in the summation alternate in sign, so the error any summation is less than that first omitted term. The final value agrees with the numerical evaluation of the integral. Both the series summation and the 9-term Simpson's rule numerical integration give solutions of $\eta_{\text {approx }}=0.66473$, differing only in the sixth significant figure.

At higher values of the Thiele modulus, the series does not always converge as well as in the above example. As an example, for the conditions $\phi=10, \eta_{\text {rigorous }}=0.067, \beta=-0.1, \varepsilon=30$, and $n=1$, the following results are obtained:

\begin{tabular}{rr}
\multicolumn{1}{c}{ term } & \multicolumn{1}{l}{ sum } \\
+0.1493 & +0.1493 \\
-0.4478 & -0.2985 \\
+1.3881 & +1.0896 \\
-4.4418 & -3.3522 \\
+14.6579 & +11.3057 \\
-49.8369 & -38.5312 \\
+174.4291 & +135.8979 \\
-627.9447 & -492.0469 \\
+2383.3952 & +1381.3484 \\
-8828.9020 & -6997.5536
\end{tabular}

Table A-1

\section{BASIC COMPUTER CODE FOR EVALUATION OF SERIES EXPANSION OF ANALYTIC SOLUTION TO INTEGRAL ON RHS OF EQ. (III-52)}

10 REM file \#c:llfblcatlysisleq52eval.bas

20 REM version of $6 / 2 / 98$

30 REM this code solves the series expansion of the analytical 40 REM solution to the integral on the RHS of Eq. III-52 in 50 REM the effectiveness factor report.

60 DEFDBL A-H,L-Z

70 DEFINT I-K

$80 \mathrm{PHI}=1$

90 BETA $=-.02$

100 EPS $=20$

$110 \mathrm{~N}=1$

120 ETARIG $=.63$

130 OMEGA $=$ ETARIG*PHI*PHI

140 ALPHA1 $=1$ - BETA*OMEGA

150 PRINT

160 PRINT " term sum"

170 PRINT 


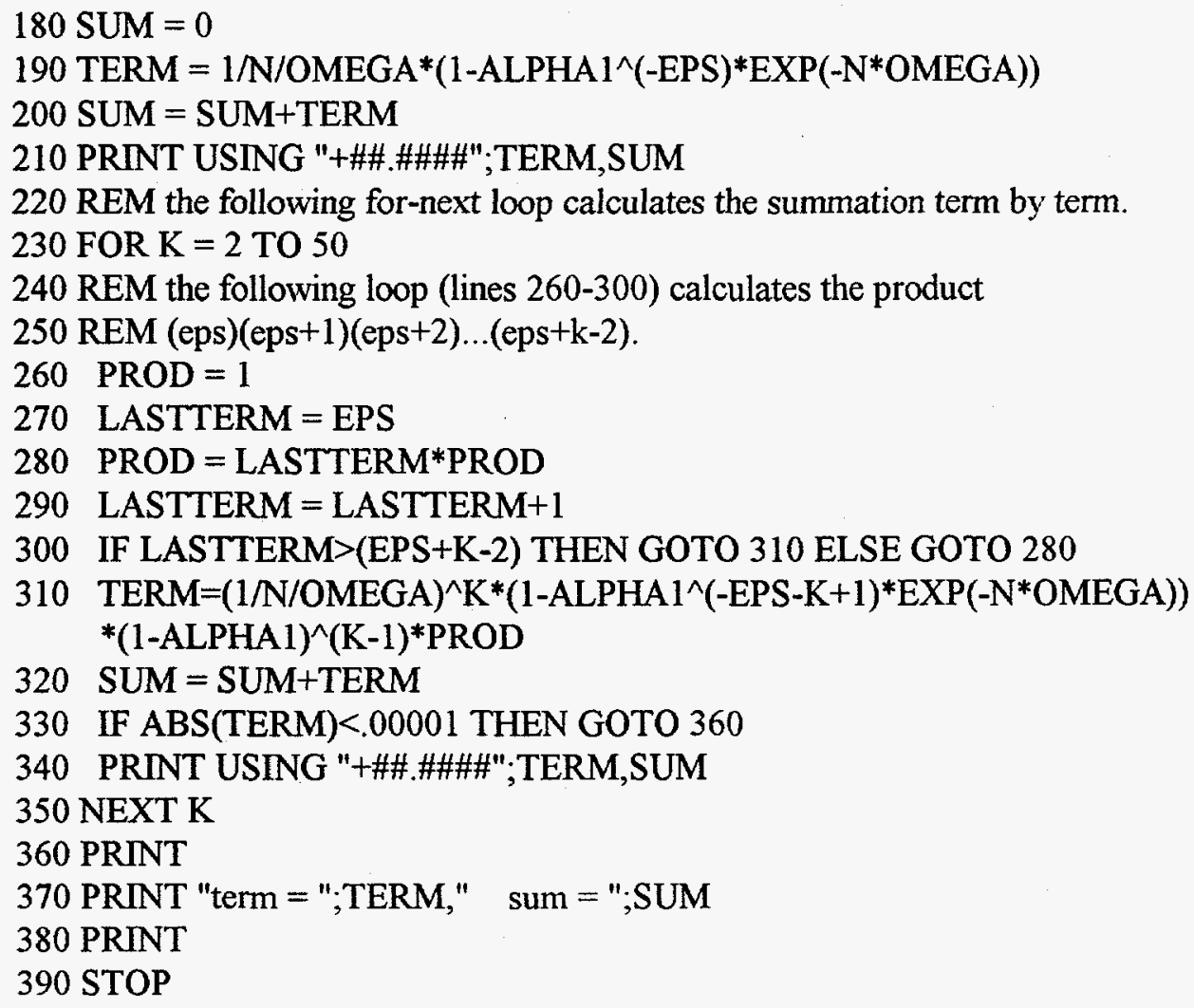




\section{APPENDIX B}

\section{LISTING OF COMPUTER CODES USED IN THIS WORK}

Table B-1

\section{BASIC COMPUTER CODE FOR ITERATIVE SOLUTION OF EQUATION (IV-5) WITH BOUNDARY CONDITIONS DEFINED BY EQUATIONS (IV-6)-(IV-7)}

10 REM file \#c: leelcatlysisleqnslv10

20 REM version of $6 / 1 / 98$

30 REM this code solves numerically the mass transfer split boundary

$40 \mathrm{REM}$ value $\mathrm{ODE}$ for a second-order reaction occurring within a porous

50 REM catalyst. the code solves the equation iteratively. an estimate

$60 \mathrm{REM}$ of the concentration (xi) at the center is made, the code calculates

70 REM the concentration profile, and the value at the boundary is

80 REM compared with the true value (one). estimates are repeated until

90 REM the code calculates a satisfactory match.

100 DEFDBL A-H,L-Z

110 DEFINT I-K

$120 \mathrm{REM} \mathrm{K}$ is 1 greater than the number of sections into which the sphere is

130 REM divided.

$140 \mathrm{~K}=10001$

150 REM PHI is the Thiele modulus.

$160 \mathrm{PHI}=1$

$170 \mathrm{DA} 2=9 * \mathrm{PHI} * \mathrm{PHI}$

180 REM DRHO is the radial difference in the numerical computation.

190 DRHO $=1 /(\mathrm{K}-1)$

200 REM DXI is the derivative of XI w.r.t. RHO at the subscripted point.

210 INPUT "xil = ";XI1

220 RHO1 $=.000001$

$230 \mathrm{DXI1}=0$

$240 \mathrm{ETA}=0$

$250 \mathrm{XI} 11=\mathrm{XI} 1$

260 REM D2XI is the derivative of DXI w.r.t. RHO.

270 REM this for-next loop calculates the concentration profile for the

280 REM spherical catalyst particle. As it does this, it also calculates

290 REM the effectiveness factor.

300 REM the trapezoidal rule is used in a predictor-corrector fashion for

310 REM calculating the values of the second derivative of the dependent

$320 \mathrm{REM}$ variable $(\mathrm{d} 2 \mathrm{xi})$. this requires values of the dependent variable

330 REM (xi) and its derivative (dxi) at the (i+1) point. these values are

340 REM obtained first by the euler method, then by iterative improvement

350 REM using the trapezoidal rule to calculate the second derivative.

360 REM when the values of the independent variable converge, the calculation

370 REM proceeds to the next step.

380 FOR I=1 TO K

390 RHO2 $=$ RHO1+DRHO 


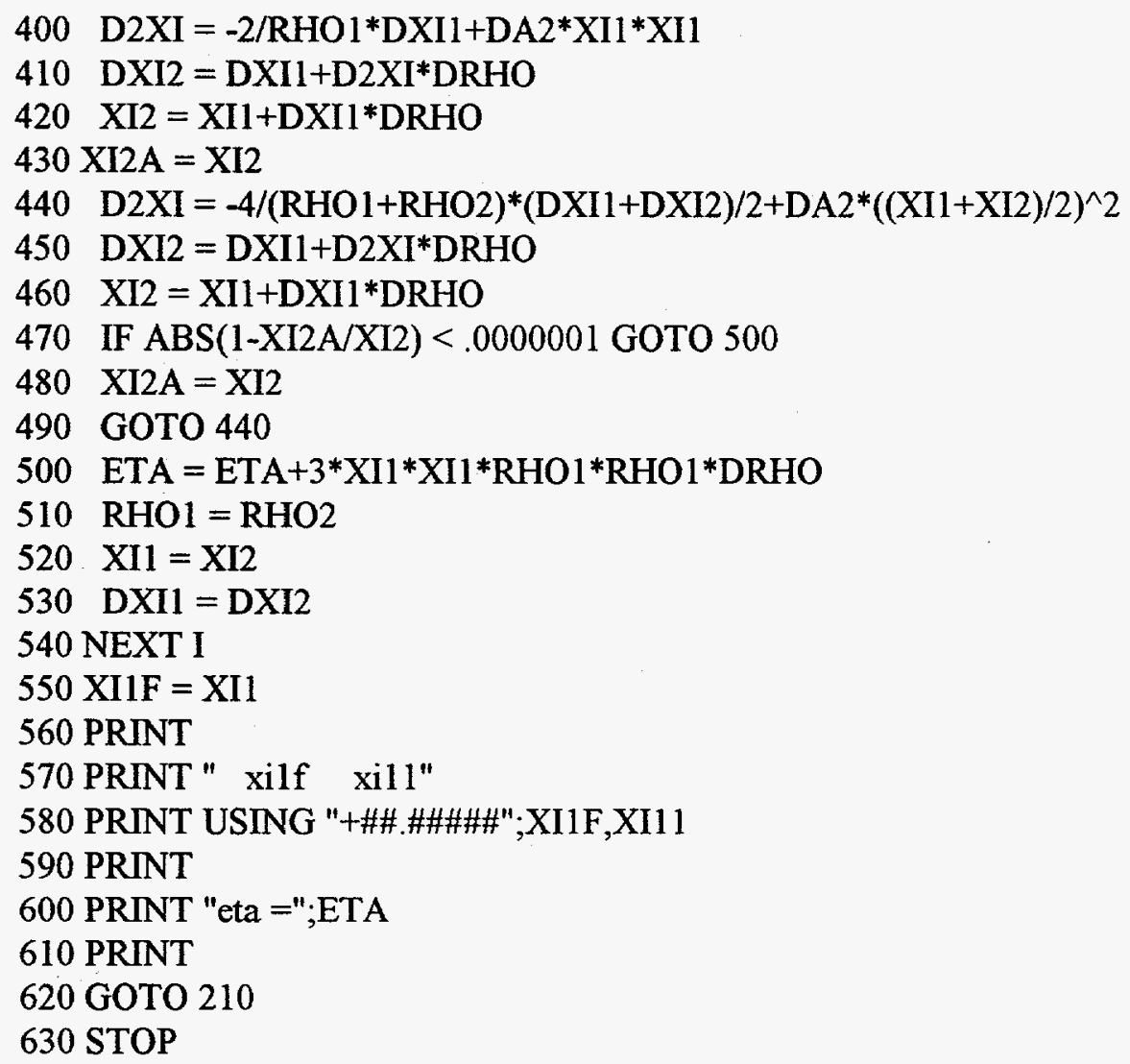


Table B-2

\section{BASIC COMPUTER CODE FOR ITERATIVE SOLUTION OF EQUATIONS (IV-15) AND (IV-16) WITH BOUNDARY CONDITIONS DEFINED BY EQUATIONS (IV-6)-(IV-7) AND (IV-17)-(IV-18)}

10 REM file \#c:llfblcatlysisleqnslv5.bas

20 REM version of $6 / 14 / 98$

30 REM this code solves numerically the simultaneous heat and mass transfer

40 REM split boundary value ODEs for a first-order reaction occurring within a

50 REM spherical porous catalyst particle. an estimate is first made of the

60 REM temperature profile. using this estimate, the code solves the equation

70 REM for the concentration profile. using the concentration profile, the code

80 REM solves the equation for the temperature profile. the new temperature

$90 \mathrm{REM}$ profile is then used in solving for a new concentration profile. the

100 REM cycle is repeated until satisfactory solutions are obtained. each of

110 REM the above steps involves iterative calculations. for example, an

120 REM estimate of the concentration (xi) at the center is made, the code

130 REM calculates the concentration profile, and the value at the boundary is

140 REM compared with the true value (one). estimates are repeated until the

150 REM calculates a satisfactory match. the analogous approach is used in

160 REM calculating the temperature (theta) profile.

170 DEFINT I-K

180 DEFDBL A-H, L-Z

190 REM K is 1 greater than the number of sections into which the sphere is

200 REM divided.

$210 \mathrm{~K}=1001$

220 DIM RHO $(\mathrm{K}+1)$, THETA $(\mathrm{K}+1), \mathrm{XI}(\mathrm{K}+1)$, DTHETA $(\mathrm{K}+1), \mathrm{DXI}(\mathrm{K}+1)$

$230 \mathrm{REM} E$ is the reaction's activation energy, $R$ is the universal gas constant

$240 \mathrm{REM}$ T0 is the temperature at the exterior surface of the catalyst.

250 REM EPS $=\mathrm{E} / \mathrm{R} / \mathrm{T} 0$.

$260 \mathrm{EPS}=30$

270 REM PHI is the Thiele modulus.

$280 \mathrm{PHI}=10$

290 REM BETA is the dimensionless maximum adiabatic change.

300 BETA $=-.1$

310 REM DA2 and DA4 are the Damkoehler numbers 2 and 4, respectively.

$320 \mathrm{DA} 2=9 * \mathrm{PHI} * \mathrm{PHI}$

330 DA4 $=$ BETA $*$ DA2

340 REM a preliminary estimate is now made of the temperature profile.

350 RHO $(1)=.000001$

360 REM DRHO is the radial difference in the numerical computation.

$370 \mathrm{DRHO}=1 /(\mathrm{K}-1)$

380 FOR I $=1$ TO K

390 THETA(I) $=.95+.05 * \mathrm{RHO}^{\wedge} 3$

400 RHO $=$ RHO + DRHO

410 NEXT I

420 REM DXI is the derivative of XI w.r.t. RHO at the subscripted point. 
$430 \mathrm{DXI}(1)=0$

440 INPUT "xil = "; XI1

$450 \mathrm{XI}(1)=\mathrm{XI} 1$

460 REM D2XI is the derivative of DXI w.r.t. RHO at the subscripted point.

470 REM this for-next loop calculates the concentration profile for the

480 REM spherical catalyst particle.

490 FOR I $=1$ TO K

500 D2XI $=-2 /$ RHO(I) ${ }^{*} \mathrm{DXI}(\mathrm{I})+\mathrm{DA} 2 * \operatorname{EXP}\left(-\mathrm{EPS}^{*}(1 / \mathrm{THETA}(\mathrm{I})-1)\right) * \mathrm{XI}(\mathrm{I})$

$510 \quad \mathrm{XI}(\mathrm{I}+1)=\mathrm{XI}(\mathrm{I})+\mathrm{DXI}(\mathrm{I}) * \mathrm{DRHO}$

$520 \mathrm{DXI}(\mathrm{I}+1)=\mathrm{DXI}(\mathrm{I})+\mathrm{D} 2 \mathrm{XI} * \mathrm{DRHO}$

530 RHO $(\mathrm{I}+1)=\mathrm{RHO}(\mathrm{I})+\mathrm{DRHO}$

540 NEXT I

$550 \mathrm{XI1F}=\mathrm{XI}(\mathrm{K})$

560 REM the following printout gives the initial estimates and the final values

570 REM of both dimensionless concentrations and temperatures.

580 PRINT

590 PRINT" xilf xill thetalf thetal"

600 PRINT USING "+\#\#.\#\#\#\#"; XI1F, XI1, THETA1F, THETA1

610 PRINT

$620 \mathrm{ETA}=0$

630 REM this for-next loop calculates the effectiveness factor.

640 FOR I $=1$ TO K

$650 \mathrm{ETA}=\mathrm{ETA}+3 * \operatorname{EXP}(-\mathrm{EPS} *(1 / \mathrm{THETA}(\mathrm{I})-1)) * \mathrm{XI}(\mathrm{I}) * \mathrm{RHO}(\mathrm{I})^{\wedge} 2 * \mathrm{DRHO}$

660 NEXT I

670 PRINT

680 PRINT "eta ="; ETA

690 PRINT

700 REM the next command automatically requires a new estimate of the center

710 REM value of the dimensionless concentration since the final value is

720 REM not close to the required value of one.

730 IF ABS(XI(K)-1) > .01 THEN GOTO 440

740 REM the next series of commands gives a choice of what to do--a choice of

750 REM " 1 " asks the operator to calculate new values of dimensionless

760 REM concentrations. a choice of " 2 " asks the operator to calculate new

770 REM values of dimensionless temperatures. a choice of " 3 " terminates the

780 REM calculations.

790 INPUT "ichoice $=$ "; ICHOICE

800 IF ICHOICE $=1$ THEN GOTO 430

810 IF ICHOICE $=2$ THEN GOTO 880

820 IF ICHOICE $=3$ THEN GOTO 1320

830 REM the next two commands trap two classes of input typographical errors.

840 IF ICHOICE $<1$ THEN GOTO 790

850 IF ICHOICE $>3$ THEN GOTO 790

860 GOTO 430

870 REM DTHETA is the derivative of THETA w.r.t. RHO at the subscripted point.

880 DTHETA $(1)=0$

890 INPUT "thetal $=$ "; THETA1

900 THETA(1) = THETA 1

910 REM D2THETA is the derivative of DTHETA w.r.t. RHO at the subscripted point. 
920 REM this for-next loop calculates the temperature profile for the spherical

930 REM catalyst particle.

940 FOR I $=1$ TO K

950 D2THETA $=-2 /$ RHO(I)*DTHETA(I)-DA4*EXP(-EPS*(1/THETA(I)-1) $) *$ XI(I)

960 THETA $(\mathrm{I}+1)=$ THETA $(\mathrm{I})+\mathrm{DTHETA}(\mathrm{I}) *$ DRHO

970 DTHETA(I+1) = DTHETA(I)+D2THETA*DRHO

980 RHO $(\mathrm{I}+1)=$ RHO $(\mathrm{I})+\mathrm{DRHO}$

990 NEXT I

1000 THETA $1 F=$ THETA(K)

1010 REM the following printout gives the initial estimates and the final values

1020 REM of both dimensionless temperatures and concentrations.

1030 PRINT

1040 PRINT " thetalf thetal xilf xil"

1050 PRINT USING "+\#\#.\#\#\#\#"; THETA1F, THETA1, XI1F, XI1

1060 PRINT

$1070 \mathrm{ETA}=0$

1080 REM this for-next loop calculates the effectiveness factor.

1090 FOR $\mathrm{I}=1 \mathrm{TO} \mathrm{K}$

1100 ETA $=$ ETA $+3 * \operatorname{EXP}\left(-E P S *(1 / \text { THETA(I)-1) })^{*} \mathrm{XI}(\mathrm{I}) * \mathrm{RHO}(\mathrm{I})^{\wedge} 2 * \mathrm{DRHO}\right.$

1110 NEXT I

1120 PRINT

1130 PRINT "eta ="; ETA

1140 PRINT

1150 REM the next command automatically requires a new estimate of the center

1160 REM values of the dimensionless temperature since the final value is

1170 REM not close to the required value of one.

1180 IF ABS(THETA(K)-1)>.01 THEN GOTO 880

1190 REM the next series of commands gives a choice of what to do--a choice of

1200 REM " 1 " asks the operator to calculate new values of dimensionless

1210 REM concentrations. a choice of "2" asks the operator to calculate new

1220 REM values of dimensionless temperatures. a choice of " 3 " terminates the

1230 REM calculations.

1240 INPUT "ichoice = "; ICHOICE

1250 IF ICHOICE $=1$ THEN GOTO 440

1260 IF ICHOICE $=2$ THEN GOTO 880

1270 IF ICHOICE $=3$ THEN GOTO 1320

1280 REM the next two commands trap two classes of input typographical errors.

1290 IF ICHOICE $<1$ THEN GOTO 1240

1300 IF ICHOICE $>3$ THEN GOTO 1240

1310 GOTO 880

1320 PRINT "the program is terminated."

1330 STOP 


\section{LITERATURE CITED}

Aris, R., "A Normalization for the Thiele Modulus," Ind. Eng. Chem. Fundam., 4, 227-229, 487 (1965).

Aris, R., Elementary Chemical Reactor Analysis, p. 135. Prentice-Hall, Englewood Cliffs, NJ, 1969.

Aris, R., The Mathematical Theory of Diffusion and Reaction in Permeable Catalysts. Oxford University Press, London, 1975.

Bischoff, K. B., "Effectiveness Factors for General Reaction Rate Forms," AIChE J., 11, 351-355 (1965).

Bischoff, K. B., "An Extension of the General Criterion for Importance of Pore Diffusion with Chemical Reactions," Chem. Eng. Sci., 22, 525-530 (1967).

Brown, L. F., "Tests for Absence of Intraparticle Diffusion Effects in Branched-Pore Systems," Chem. Eng. Sci., 27, 213-219 (1972).

Carberry, J. J., Chemical and Catalytic Reaction Engineering, pp. 224 (Fig. 5-11), 227-228, 232. McGraw-Hill, New York, 1976.

Catchpole, J. P., and G. Fulford, "Dimensionless Groups," Ind. Eng. Chem., 58(3), 46-60 (March, 1966).

Gonzo, E. E., and J. C. Gottifredi, "Nonisothermal Effectiveness Factor Estimation with Simple Analytical Expressions," Lat. Am. J. Heat Mass Transfer, 6, 113-129 (1982) (as cited in Haynes 1986).

Gonzo, E. E., and J. C. Gottifredi, "General Diagnostic Criteria for Transport Limitation in Porous Solid Chemical Reactions," J. Catal., 83, 25-31 (1983a).

Gonzo, E. E., and J. C. Gottifredi, "Rational Approximations of Effectiveness Factor and General Diagnostic Criteria for Heat and Mass Transport Limitations," Catal. Rev.—Sci. Eng., 25, 119-140 (1983b).

Gonzo, E. E., J. C. Gottifredi and L. C. Romero, "Effectiveness Factors and Selectivity for Parallel Catalytic Reactions with Langmuir-Hinshelwood Kinetics," Chem. Eng. Sci., 43, 1410-1413 (1988).

Goto, S., and H. Ito, "Approximate Solutions of Effectiveness Factor Based on Bulk Conditions," Can. J. Chem. Eng., 68, 167-170 (1990).

Gottifredi, J. C., E. E. Gonzo and O. D. Quiroga, "Isothermal Effectiveness Factor-I. Analytical Expression for Single Reaction with Arbitrary Kinetics, Slab Geometry," Chem. Eng. Sci., 36, 706-711 (1981a).

Gottifredi, J. C., E. E. Gonzo and O. D. Quiroga, "Isothermal Effectiveness Factor-II. Analytical Expression for Single Reaction with Arbitrary Kinetics, Geometry and Activity Distribution," Chem. Eng. Sci., 36, 713-719 (1981b).

Haynes, H. W., Jr., "An Explicit Approximation for the Effectiveness Factor in Porous Heterogeneous Catalysts," Chem. Eng. Sci., 41, 412-415 (1986).

Hudgins, R. R., "A General Criterion for Absence of Diffusion Control in an Isothermal Catalyst Pellet," Chem. Eng. Sci., 23, 93-94 (1968).

Kubota, H., and Y. Yamanaka, "Remarks on Approximate Estimation of Catalyst Effectiveness Factor," $J$. Chem. Eng. Jap., 2, 238-240 (1969).

Liaw, C. H., J. S. P. Wang, R. A. Greenkorn and K. C. Chao, "Kinetics of Fixed-Bed Adsorption: a New Solution," AIChE J., 25, 376-381 (1979). 
Petersen, E. E., Chemical Reaction Analysis, pp. 64-66. Prentice-Hall, Englewood Cliffs, NJ, 1965.

Rice, R. G., "Approximate Solution for Batch, Packed Tube and Radial Flow Adsorption-Comparison with Experiment," Chem. Eng. Sci., 37, 83-91 (1982).

Roberts, G. W., and C. N. Satterfield, "Effectiveness Factor for Porous Catalysts," Ind. Eng. Chem. Fundam., 4, 288-293 (1965).

Stewart, W. E., and J. E. Villadsen, "Graphical Calculation of Multiple Steady States and Effectiveness Factors for Porous Catalysts," AIChE J., 15, 28-34 (1969).

Suenson, M. M., C. Georgakis and T. W. Root, "A Semi-Analytical Approximation to the Effectiveness Factor for a Non-Linear Isothermal Reaction and Diffusion Problem," Chem. Eng. Sci., 38, 188-489 (1983).

Tinkler, J. D., and A. B. Metzner, "Reaction Rates in Nonisothermal Catalysts," Ind. Eng. Chem., 53(8), 663-668 (Aug., 1961).

Wedel, S., and D. Luss, "A Rational Approximation of the Effectiveness Factor," Chem. Eng. Comm., 7, 245-259 (1980).

Weisz, P. B., and C. D. Prater, "Interpretation of Measurements in Experimental Catalysis," Adv. Catalysis, 6, 143-196, esp. 167-168 (1954).

Weisz, P. B., and J. S. Hicks, "The Behavior of Porous Catalyst Particles in View of Internal Mass and Heat Diffusion Effects," Chem. Eng. Sci., 17, 265-275 (1962).

Xiu, G.-h., T. Nitta, P. Li and G. Jin, "Breakthrough Curves for Fixed-Bed Adsorbers-Quasi-Log Normal Distribution Approximation," AIChE J., 43, 979-985 (1997).

Yin, Q. X., and S. F. Li, "Rational Approximation of the Overall Effectiveness Factor for the Gas-LiquidSolid Phase Catalytic Reaction," Ind. Eng. Chem. Res., 34, $3771-3776$ (1995).

Yin, Q. X., and S. F. Li, "Approximate Analytical Solutions of Effectiveness Factors for Parallel Catalytic Reactions," Chem. Eng. J., 59, 161-167 (1995). 